\title{
A physical-space version of the stretched-vortex subgrid-stress model for large-eddy simulation
}

\author{
Tobias Voelkl and D. I. Pullin \\ Graduate Aeronautical Laboratories 301-46, California Institute of Technology, Pasadena, California 91125 \\ Daniel C. Chan \\ GE Corporate Research and Development Center, Room KW-C118, One Research Circle, Niskayuna, \\ New York 12309
}

(Received 15 July 1999; accepted 14 March 2000)

\begin{abstract}
A physical-space version of the stretched-vortex subgrid-stress model is presented and applied to large-eddy simulations of incompressible flows. This version estimates the subgrid-kinetic energy required for evaluation of the subgrid-stress tensor using local second-order structure-function information of the resolved velocity field at separations of order the local cell size. A relation between the structure function and the energy spectrum is derived using the kinematic assumptions of the stretched-vortex model for locally homogeneous anisotropic turbulence. Results of large-eddy simulations using this model are compared to experimental and direct numerical simulation data. Comparisons are shown for the decay of kinetic energy and energy spectra of decaying isotropic turbulence and for mean velocities, root-mean-square velocity fluctuations and turbulence-kinetic energy balances of channel flow at three different Reynolds numbers. (C) 2000 American Institute of Physics. [S1070-6631(00)00207-5]
\end{abstract}

\section{INTRODUCTION}

Large-eddy simulation (LES) was introduced as an approach to surmount some of the obstacles to the prediction of turbulent flows by direct numerical simulation (DNS). The DNS of the Navier-Stokes equations for fully turbulent flows at large Reynolds numbers requires resolution capabilities outside the range of computers in the present and the foreseeable future. In LES, scales at wave numbers smaller than some cutoff, say $k=k_{c}$ ( $k$ is the wave number), are computed directly while the subgrid scales and their principal physical effect on these large or resolved scales, that of providing an energy sink via energy cascade to the dissipation-range scales, are represented by subgrid-stress (SGS) models. The underlying assumption is that these subgrid scales do not depend strongly on the particular geometry of the flow and that therefore models applicable to a wide range of flows can be developed. The main issue in SGS modeling is the formulation of a tractable but physically realistic description of the dynamical interaction between the resolved scales and the subgrid scales. The latter may be termed the "fine scales" of the turbulence. For present purposes they may be taken to extend from somewhere within the inertial range (at large Reynolds numbers) down to the Kolmogorov scale $\eta=\left(\nu^{3} / \epsilon\right)^{1 / 4}$ where $\nu$ is the viscosity and $\epsilon$ the local dissipation.

Subgrid-stress models must be robust across many different types of flow including free and wall-bounded turbulence, transition flows, separated flows and bluff body flows, and should in principle be free of flow-dependent adjustable parameters. They must be capable of operating efficiently and accurately at spatial resolutions very substantially less than those required for full DNS and with numerical methods that can be applied to flows with complex boundary condi- tions. Most importantly, models should provide a rational framework for extension to mixing flows including the transport of passive and active scalars, combusting flows and strongly compressible flows. There are many different approaches to this task-see Refs. 1 and 2 for reviews. The eddy-viscosity ansatz is the earliest and remains the most widely used basis for SGS modeling. Smagorinsky's model for the eddy-viscosity, originally developed for atmospheric turbulence, has been applied extensively to engineering applications. The formulation with a fixed model constant has well-known shortcomings for wall-bounded flow and this has been largely superseded by dynamic procedures to determine the Smagorinsky constant based on double filtering. ${ }^{3,4}$ Some mathematical inconsistencies which arise in the dynamical formulation can be resolved by casting the problem in variational form, resulting in the dynamic localization model. ${ }^{5}$ In applications, simplified solutions to the difficulties arising in the dynamic procedure are widely used, for example plane averaging in homogeneous directions ${ }^{3}$ or approximate localization. ${ }^{6}$

A related class of SGS models are the spectral eddyviscosity models. ${ }^{7,8}$ This approach was extended to operate in physical space through the first use of velocity structure functions $^{7}$ as a substitute for the spectral information required to estimate the eddy viscosity. For use in large-eddy simulations, ${ }^{9,10}$ this model has been combined with additional filtering or a switch, which selectively turns the eddy viscosity on and off. ${ }^{1}$

A different approach was proposed by Leonard, ${ }^{11,12}$ based on retaining the first term(s) of a formal series which expresses the result of filtering a product of two quantities solely in terms of products of the gradients of filtered quantities, thereby theoretically providing an exact closure for the 
(Gaussian) filtered LES equations. This is often termed the gradient model. As described in Ref. 12, special regularization techniques (e.g., using particle methods) may have to be used in the numerical methods implementing this model. Alternatively the gradient model could be included in mixed models, for example with an eddy-viscosity term, as in the nonlinear model of Kosović. ${ }^{13}$ A related idea, namely to invert the filtering operation, augmented by a procedure to generate a range of subgrid scales on a finer mesh, leads to the subgrid-scale estimation model, ${ }^{14}$ which was originally developed in spectral space, but can also be applied in physical space. ${ }^{15,16}$ The present paper describes continuing work on a different class of models, the so-called stretched-vortex SGS models.

We will consider incompressible, constant-density flow. The usual starting point for LES are the resolved-scale Navier-Stokes equations

$$
\begin{aligned}
& \frac{\partial \widetilde{U}_{i}}{\partial x_{i}}=0, \\
& \frac{\partial \widetilde{U}_{i}}{\partial t}+\frac{\partial}{\partial x_{j}}\left(\widetilde{U}_{i} \widetilde{U}_{j}\right)=-\frac{1}{\rho} \frac{\partial \widetilde{P}}{\partial x_{i}}-\frac{\partial \tau_{i j}}{\partial x_{j}}+\nu \frac{\partial^{2} \widetilde{U}_{i}}{\partial x_{j} \partial x_{j}},
\end{aligned}
$$

where $\widetilde{U}_{i}$ is a resolved-scale velocity field and $\tau_{i j}$ is the subgrid-stress (SGS) tensor, which represents the effect of subgrid scales on the resolved flow. Equations (1) and (2) can be derived formally by applying a filtering operator to the full Navier-Stokes equations. ${ }^{11}$ This filtering operation to obtain the resolved-scale velocity field cannot be performed explicitly in actual LES. Instead, the resolved field is the result of the numerical integration of Eqs. (1) and (2) using a given numerical method on a grid with a given resolution whose smallest scale is much larger than the smallest turbulence scale. This requires a model which expresses $\tau_{i j}$ as a functional of the resolved-scale velocity field $\widetilde{U}_{i}$. This is the SGS model.

One approach to the construction of SGS models is to apply certain formal properties of the filtering operation ${ }^{3,17}$ to all physical quantities appearing in (2). Presently we follow a different path by considering a model which is motivated by vortex/structure-based descriptions of the fine scales of turbulence. Our aim is to construct a local model which approximates the effect of the subgrid motion on the resolved scales in a physical way. We will not attempt to either interpret or to analyze this model using elements of the filtering operation. Further, our goal is to obtain estimates for the low-order statistics of a full turbulent field which can be compared directly to results of unfiltered DNS or experimental measurement. This will usually involve an estimate of subgrid contributions to turbulence transport properties. There will be exceptions to this, which will be noted in the sequel. In summary, we seek to calculate the turbulence itself, for which the component filtered to the resolution of the numerical method may be dominant but is not complete. In this sense our approach might be described as highly under resolved turbulence simulation (HURTS). We will attempt to follow the same approach in analyzing the turbulence transport budget.
Our model development is based on kinematics of homogeneous anisotropic turbulence generated by simple vortex structures. The resulting stretched-vortex SGS model in its present form for incompressible flows is described in Sec. II. In Sec. II A, previous work on this model is briefly reviewed, and the new developments for a physical-space version are presented in Sec. II B. Some comments on numerical methods and model implementation are made in Sec. III. The results of the large-eddy simulations, in which the new model has been tested, are presented in Sec. IV.

\section{A PHYSICAL-SPACE VERSION OF THE STRETCHED-VORTEX SUBGRID-STRESS MODEL}

\section{A. Review of the stretched-vortex model}

A class of vortex-based models for the subgrid-stress tensor has been proposed by Pullin and Saffman ${ }^{18}$ (henceforth denoted by PS). Locally straight and nearly axisymmetric vortex structures are used to model the small-scale vorticity. These subgrid elements are vortices in the sense that they provide subgrid motion only in a plane normal to the vortex axis. Finite-length effects of these "vortices" are ignored, as is any possible axial motion in the vortex cores. The proposed subgrid structure is extremely simple. When combined with the stretching strain provided by the local resolved flow, it may nevertheless contain sufficient of the cascade physics to provide a viable model of the transfer from resolved to subgrid scales.

Based on this model assumption, PS derive a kinematic result for the two-point velocity correlation tensor for homogeneous anisotropic turbulence. Assuming local homogeneity for the unresolved scales in a large-eddy simulation, this result can be used to model $\tau_{i j}$ of (2) as

$$
\tau_{i j}=2 \int_{k_{c}}^{\infty} E(k) d k\left\langle\mathrm{E}_{p i} \mathrm{Z}_{p q} \mathrm{E}_{q j}\right\rangle,
$$

where $k_{c}$ is the cutoff wave number, $E(k)$ is the subgridenergy spectrum, $E_{i j}$ is the transformation matrix from vortex-fixed to laboratory axes, and $Z_{i j}$ is a diagonal matrix with diagonal elements $\left(\frac{1}{2}, \frac{1}{2}, 0\right)$. Equation (3) is the starting point for the stretched-vortex SGS model for LES. The angle brackets in (3) denote the expectation of a function $f\left(\mathrm{E}_{i j}\right)$ averaged over the orientations of the vortex structures. The orientation is described by the probability density function (pdf) $P(\alpha, \beta)$ of the Euler angles $\alpha$ and $\beta$, which represent the transformation from the vortex-fixed axes to the laboratory axes. The orientation average is then given by

$$
\left\langle f\left(\mathrm{E}_{i j}\right)\right\rangle=\frac{1}{4 \pi} \int_{0}^{\pi} \int_{0}^{2 \pi} f\left(\mathrm{E}_{i j}\right) P(\alpha, \beta) \sin \alpha d \alpha d \beta .
$$

It is assumed that $P$ does not depend on the third Euler angle, the spin angle $\gamma$ about the vortex axis. This does not limit the internal vorticity distribution of the vortex structures to axisymmetric cases. Equation (3) holds for an arbitrary vorticity distribution in an individual structure following averaging over $\gamma$, when $\gamma$ is uniformly distributed in $0 \leqslant \gamma \leqslant 2 \pi$. Knowledge of the internal vorticity distribution beyond the assumptions stated above is not required in this model. 
In order to use Eqs. (3) and (4) for a viable SGS model, two more steps are necessary. First, the pdf of the Euler angles $P(\alpha, \beta)$ needs to be determined, and second, an estimate for the subgrid-kinetic energy

$$
K \equiv \int_{k_{c}}^{\infty} E(k) d k
$$

is required. Misra and Pullin, ${ }^{19}$ henceforth referred to as MP, used a pdf given by the product of delta functions or a linear combination of such products of delta functions of the type

$$
P(\alpha, \beta)=\frac{4 \pi}{\sin \alpha} \delta(\alpha-\theta) \delta(\beta-\phi),
$$

where $\theta(\mathbf{x}, t), \phi(\mathbf{x}, t)$ are particular Euler angles. Defining the unit vector $\mathbf{e}_{v}$ of the vortex axis with components

$$
e_{1}^{v}=\sin \theta \cos \phi, \quad e_{2}^{v}=\sin \theta \sin \phi, \quad e_{3}^{v}=\cos \theta,
$$

Eq. (3) can then be written in the form

$$
\tau_{i j}=K\left(\delta_{i j}-e_{i}^{v} e_{j}^{v}\right) .
$$

Misra and Pullin ${ }^{19,20}$ tested several particular models for $P(\alpha, \beta)$ including alignment with eigenvectors of the resolved rate-of-strain tensor, alignment with the resolved vorticity vector and a model in which the vortex alignment responded kinematically to the time-varying velocity-gradient tensor of the resolved field. Of these, the simplest model assumes that a single subgrid vortex aligns, with weighted probability, with the eigenvectors corresponding to the maximum extensional and intermediate eigenvalues of the resolved rate-of-strain tensor.

To estimate $K$, MP assume a Kolmogorov form of $E(k)$ with a sharp viscous cutoff

$$
\begin{aligned}
E(k) & =\mathcal{K}_{0} \epsilon^{2 / 3} k^{-5 / 3}, \quad k_{c}<k<J \eta^{-1} \\
& =0, \quad k>J \eta^{-1},
\end{aligned}
$$

where $\mathcal{K}_{0}$ is the Kolmogorov prefactor, $\eta=\left(\nu^{3} / \epsilon\right)^{1 / 4}$ is the "local" Kolmogorov length, and $J$ is a cutoff parameter. The concept of a local Kolmogorov length and accordingly a local dissipation $\epsilon$ has to be understood in the LES context: A gridcell in a large-eddy simulation will typically be large compared to the smallest scales in turbulence, and therefore the turbulent motions in one grid cell can be viewed as their own realization or statistical sample of turbulence, which can be described (approximately) by its own statistical quantities. We also emphasize that for stretched-vortex models of the fine scales, $E(k)$ depends only on the internal structure of the vorticity and is independent of $P .{ }^{18}$ This somewhat surprising result can be understood physically using the well-known relation for homogeneous nonisotropic turbulence, $E(k)$ $=E_{\omega}(k) /\left(2 k^{2}\right)$, where $E_{\omega}$ is the vorticity spectrum. It is clear that for a field with straight, parallel vortex lines (present rectilinear vortex model), the trace of the two-point vorticity correlation tensor must be independent of the orientation of these lines. It then follows that $E_{\omega}(k)$, and hence $E(k)$ must be independent of the vortex orientation. Thus there is no assumption of local isotropy and therefore no inconsistency in combining a Kolmogorov spectrum with local anisotropy. Alternatives to the sharp dissipation cutoff of
(9) would be to assume that each subgrid vortex is of the (nearly axisymmetric) stretched-spiral form, and to replace (9) with the Lundgren ${ }^{21}$ spectrum, or to use an exponential cutoff near $k \eta=1$ as suggested by $\mathrm{DNS}^{22}$ and experiment. ${ }^{23}$ The sharp cutoff is chosen for simplicity. The quantity $J$ is a model parameter. A cutoff at the Kolmogorov scale $k_{c} \eta$ $=1(J=1)$ is preferred on physical grounds but there is some analytical simplification if one lets $J \rightarrow \infty$.

Equation (9) contains as unknowns $\epsilon$ and $\mathcal{K}_{0}$. MP estimated these by first using the assumption of a local balance between the total dissipation $\epsilon$ on one side and the sum of the resolved-scale dissipation and the subgrid "dissipation" $\varepsilon_{\mathrm{sgs}}$ on the other side

$\epsilon=2 \nu \widetilde{S}_{i j} \widetilde{S}_{i j}+\varepsilon_{\mathrm{sgs}}, \quad \varepsilon_{\mathrm{sgs}} \equiv-\widetilde{S}_{i j} \tau_{i j}=-K \widetilde{S}_{i j}\left(\delta_{i j}-e_{i}^{v} e_{j}^{v}\right)$.

More precisely, $\varepsilon_{\mathrm{sgs}}$ represents the production of subgridkinetic energy by the interaction between the resolved rateof-strain tensor and the subgrid stresses, and its transfer from resolved to subgrid scales. In using (10) it is implicitly assumed that this energy is dissipated within the local subgrid motion, and is not transferred elsewhere. This is consistent with an algebraic, zero-equation SGS model. In their dynamic version of the stretched-vortex model, MP used (10) and a continuity condition between resolved and subgrid spectra to obtain a (global) system of equations which could be solved for $\epsilon$ at each gridpoint and a global $\mathcal{K}_{0}$, thus allowing the SGS tensor to be computed from the resolvedscale quantities. For channel flow this matching was done, with appropriate kinematics, in planes defined by the two directions of flow homogeneity. This required the solution of coupled systems of equations for the $\epsilon$ field.

\section{B. Localized estimation of the subgrid-kinetic energy in physical space}

In the following, we describe a version of the stretchedvortex model that uses only local information. This version does not require the knowledge of a resolved-scale energy spectrum, nor the solution of a coupled system of equations, and works entirely in physical space (as opposed to Fouriertransform space). The vortex orientation $\mathbf{e}_{v}$ is determined in the usual way, in the present work by using alignment models of the form given by (6). But the subgrid-kinetic energy, the other quantity required to evaluate the model equation for the SGS tensor $\tau_{i j}$, is estimated in a new way.

\section{Structure-function relation for the stretched-vortex model with delta-function pdf}

The present version makes use of the relation between the energy spectrum in Fourier space and the second-order structure functions in physical space (see, e.g., Batchelor ${ }^{24}$ ). The use of second-order structure functions for the estimation of SGS parameters in LES was first reported by Métais and Lesieur, ${ }^{7}$ who used the relation for isotropic turbulence.

We now derive a structure-function relation based on the kinematics of the stretched-vortex model. The starting point is an expression for the diagonal components of the onedimensional spectrum tensor $\Theta_{i j}\left(k_{3}\right)$, Eqs. (49), (50), and 
(51) of PS. Taking the trace of $\Theta_{i j}\left(k_{3}\right)$, substituting the pdf (6), and performing the integration over the Euler angles $\alpha$, $\beta$ yield:

$$
\begin{aligned}
& \Theta_{11}\left(k_{3}\right)+\Theta_{22}\left(k_{3}\right)+\Theta_{33}\left(k_{3}\right) \\
& =\frac{2}{\pi} \int_{\left|k_{3} / \sin \theta\right|}^{\infty} E(\kappa)\left(\kappa^{2}-\frac{k_{3}^{2}}{\sin ^{2} \theta}\right)^{-1 / 2} \frac{d \kappa}{\sin \theta} .
\end{aligned}
$$

In (11), $k_{3}$ is the three-axis wave number and $\theta$, as in (7), is the angle between the three-axis (laboratory frame) and the (local) orientation of the subgrid-vortex structure. Applying an inverse Fourier transform to the last equation, we obtain the trace of the velocity correlation tensor $R_{i j}\left(0,0, r_{3}\right)$ for a separation $r_{3}$ in the three-direction of the coordinate system

$$
\begin{aligned}
R_{11}+R_{22}+R_{33}= & \frac{2}{\pi} \int_{k_{3}=-\infty}^{\infty} \int_{\kappa=\left|k_{3} / \sin \theta\right|}^{\infty} E(\kappa) \\
& \times\left(\kappa^{2}-\frac{k_{3}^{2}}{\sin ^{2} \theta}\right)^{-1 / 2} \frac{d \kappa}{\sin \theta} e^{i k_{3} r} d k_{3} .
\end{aligned}
$$

After interchanging the order of integration, the integral over $k_{3}$ can be evaluated analytically, and one obtains $\left(r \equiv r_{3}\right)$

$$
R_{11}+R_{22}+R_{33}=2 \int_{0}^{\infty} E(\kappa) J_{0}(r \kappa \sin \theta) d \kappa,
$$

where $J_{0}$ is the Bessel function of the first kind. Next we use that, with $R_{i j}=\overline{U_{i} \hat{U}_{j}}$, where $U_{i}$ and $\hat{U}_{i}$ are velocity components at points with coordinates $\mathbf{x}$ and $\hat{\mathbf{x}}=\mathbf{x}+r \mathbf{e}_{3}$, respectively, and where the overline is a volume average, we can write

$$
\begin{aligned}
R_{11}+R_{22}+R_{33}= & \overline{U_{1}^{2}}+\overline{U_{2}^{2}}+\overline{U_{3}^{2}}-\frac{1}{2} \overline{\left[\left(\hat{U}_{1}-U_{1}\right)^{2}\right.} \\
& \left.+\overline{\left(\hat{U}_{2}-U_{2}\right)^{2}}+\overline{\left(\hat{U}_{3}-U_{3}\right)^{2}}\right] \\
= & 2 \int_{0}^{\infty} E(k) d k-\frac{1}{2} \mid \overline{\mathbf{U}-\left.\mathbf{U}\right|^{2}}
\end{aligned}
$$

Combining (13) and (14) we obtain a structure-function relation for the stretched-vortex model with delta-function pdf as

$$
F_{2}(\mathbf{r} ; \mathbf{x}) \equiv \overline{|\hat{\mathbf{U}}-\mathbf{U}|^{2}}=4 \int_{0}^{\infty} E(k)\left[1-J_{0}(r k \sin \theta)\right] d k
$$

We remark that for homogeneous anisotropic turbulence, $F_{2}$ is a function of the separation $\mathbf{r}$ only. In the present SGS modeling application we will use (15) in a local approximation at different points on the resolved-flow grid, and we retain the parametric dependence on $\mathbf{x}$.

To apply (15) to LES, we split the structure function of the full velocity field $F_{2}(\mathbf{r} ; \mathbf{x})$ into a resolved-scale contribution $\widetilde{F}_{2}(\mathbf{r} ; \mathbf{x})=|\hat{\mathbf{U}}-\widetilde{\mathbf{U}}|^{2}$ and a subgrid contribution $F_{2, \mathrm{sg}}(\mathbf{r} ; \mathbf{x})$

$$
F_{2}(\mathbf{r} ; \mathbf{x})=\widetilde{F}_{2}(\mathbf{r} ; \mathbf{x})+F_{2, \mathrm{sg}}(\mathbf{r} ; \mathbf{x}) .
$$
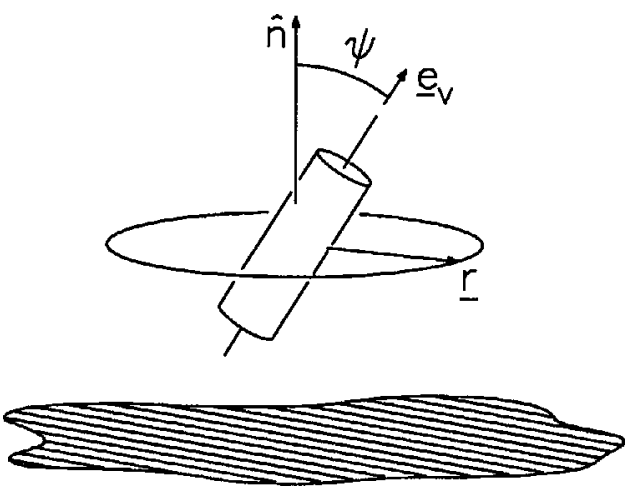

FIG. 1. Sketch of the geometry used in the circular average of the structurefunction relation for the stretched-vortex model.

The structure-function relation can be used to express $F_{2, \text { sg }}(\mathbf{r} ; \mathbf{x})$ in terms of the energy spectrum:

$$
F_{2}(\mathbf{r} ; \mathbf{x})=\widetilde{F}_{2}(\mathbf{r} ; \mathbf{x})+4 \int_{k_{c}}^{\infty} E(k)\left[1-J_{0}(r k \sin \theta)\right] d k .
$$

Substituting this in Eq. (15) and collecting the two integral terms into one, we obtain

$$
\widetilde{F}_{2}(\mathbf{r} ; \mathbf{x})=4 \int_{0}^{k_{c}} E(k)\left[1-J_{0}(r k \sin \theta)\right] d k .
$$

We recall that this relation was derived using the velocity correlation tensor for a separation $\mathbf{r}=\left(0,0, r_{3}\right)$. But for any vector $\mathbf{r}$ we can always choose a coordinate system such that the three-axis is aligned with $\mathbf{r}$. The angle $\theta$ always represents the angle between $\mathbf{r}$ and the vortex orientation $\mathbf{e}_{v}$.

\section{Model equation for $\mathcal{K}_{0} \epsilon^{2 / 3}$ using a circular average}

Equation (18) serves as our basis for estimation of SGS parameters by use of a local average over suitable directions of $\mathbf{r}$ at each gridpoint $\mathbf{x}$. This is done presently by averaging $\widetilde{F}_{2}(\mathbf{r} ; \mathbf{x})$ over a circle of radius $r=|\mathbf{r}|$ (which will depend on the local meshwidth of the computational grid) lying in a plane with normal $\hat{\mathbf{n}}$, such that $\mathbf{r}$ joins the circle center to a point on its perimeter. A sketch of the geometry is provided in Fig. 1. This is subsequently referred to as a circular average. It was chosen because it is applicable equally well to both free and wall-bounded flows, and is used for all LES reported presently. We remark that a spherical average could be used for free turbulent flows but is not suitable for nearwall flows, owing to the large shear combined with the suppression of turbulence in the sublayer. The spherical average of (18) is given in Appendix A where it is demonstrated that the well-known structure-function relation for isotropic turbulence is recovered.

Using the circular average, denoted by superscript $c$, we obtain

$$
\begin{aligned}
\widetilde{F}_{2}^{c}(r ; \mathbf{x})= & \frac{2}{\pi} \int_{\phi=0}^{2 \pi} \int_{k=0}^{k_{c}} E(k) \\
& \times\left[1-J_{0}\left(k r \sqrt{1-\sin ^{2} \psi \cos ^{2} \phi}\right)\right] d k d \phi .
\end{aligned}
$$


Here we have used the geometric relation $\cos \theta$ $=\sin \psi \cos \phi$, where $\cos \psi=\mathbf{e}^{v} \cdot \hat{\mathbf{n}}$, i.e., $\psi$ is the angle between $\mathbf{e}^{v}$ and $\hat{\mathbf{n}}$. The angle $\phi$ is the polar angle, over which the average is taken.

\section{Closure of the model}

In order to make use of the last equation, we need to assume a functional form for $E(k)$. We use the same Kolmogorov spectrum with sharp viscous cutoff as in Eq. (9).
We note that in this case the spectrum is applied over the range $0 \leqslant k \leqslant k_{c}$. But only the integral over the spectrum enters into the model, and therefore a more detailed description of the energy spectrum is not expected to be necessary for the purposes of this model. We also note that convergence of the integral at the lower limit, i.e., $k \rightarrow 0$, is guaranteed since $1-J_{0}(x)=o\left(x^{2}\right)$ for $x \rightarrow 0$. Substituting (9) into (19) and changing the integration variable to $s=k \Delta$, where $\Delta$ is the length scale at the cutoff, i.e., $k_{c}=\pi / \Delta$, we can solve for the unknown factor $\mathcal{K}_{0} \epsilon^{2 / 3}$

$$
\mathcal{K}_{0} \epsilon^{2 / 3}=\frac{\pi \widetilde{F}_{2}^{c}(r ; \mathbf{x})}{2 \Delta^{2 / 3} \int_{\phi=0}^{2 \pi} \int_{s=0}^{\pi} s^{-5 / 3}\left[1-J_{0}\left(s(r / \Delta) \sqrt{1-\sin ^{2} \psi \cos ^{2} \phi}\right)\right] d s d \phi} .
$$

In (20), $r$ and $\Delta$ are related to the local mesh size of the (possibly nonuniform) computational grid (see Sec. IIIC). All other quantities on the right-hand side can be estimated from the resolved-scale velocity field. The integral in the denominator is a function of $r / \Delta$ and $\psi$. An approximate analytical form is given in Appendix B. Methods for an effective implementation of (20) in large-eddy simulations are discussed in Sec. III C.

Equation (20) only applies for $k_{c}<J \eta^{-1}$ owing to the viscous cutoff in the model energy spectrum, Eq. (9). (We recall that $\eta$ is understood as a "local," gridcell Kolmogorov scale in LES.) In the model implementation, the cases $k_{c}<J \eta^{-1}$ and $k_{c}>J \eta^{-1}$ have to be distinguished unless a simplified form of the spectrum with a $k^{-5 / 3}$ range extending to infinity is used, $J \rightarrow \infty$. This latter choice simplifies the model significantly. In this limit Eq. (20) is valid everywhere. The group $\mathcal{K}_{0} \epsilon^{2 / 3}$ is obtained from Eq. (20), and then the (estimated) subgrid-kinetic energy follows from (9) as

$$
K=\frac{3}{2} \mathcal{K}_{0} \epsilon^{2 / 3} k_{c}^{-2 / 3} .
$$

All quantities in Eq. (8) are known, and the SGS tensor $\tau_{i j}$ can be computed.

\section{Formulation for finite $J$}

The model equations can also be closed for finite $J$ but the complexity of the resulting model version is somewhat increased compared to the $J \rightarrow \infty$ version. The local balance equation (10) is used. The subgrid-kinetic energy is now

$$
\begin{aligned}
K & =\frac{3 \mathcal{K}_{0} \epsilon^{2 / 3}}{2 k_{c}^{2 / 3}}\left[1-\left(\frac{k_{c} \eta}{J}\right)^{2 / 3}\right], \quad k_{c}<J \eta^{-1} \\
& =0, \quad k_{c}>J \eta^{-1} .
\end{aligned}
$$

Since the subgrid-kinetic energy vanishes for $k_{c}>J \eta^{-1}$, the SGS tensor will vanish accordingly. If $K=0$ for a grid cell, this means that locally all the energy is contained in the resolved scales, i.e., all relevant turbulence scales are resolved at that location, and no model contribution is needed.
Since $\eta$ is not known a priori in a cell, we first calculate the product $k_{c} \eta$ and then test for $k_{c} \eta \lessgtr J$. For $k_{c} \eta<J$, substituting $K$ from Eq. (22) into Eq. (10) yields

$$
\begin{gathered}
\epsilon=2 \nu \widetilde{S}_{i j} \widetilde{S}_{i j}-\frac{3 \mathcal{K}_{0} \epsilon^{2 / 3}}{2 k_{c}^{2 / 3}}\left[1-\left(\frac{k_{c} \eta}{J}\right)^{2 / 3}\right] \\
\times \widetilde{S}_{i j}\left(\delta_{i j}-e_{i}^{v} e_{j}^{v}\right), \quad k_{c} \eta<J .
\end{gathered}
$$

This can be written as

$$
1-\Pi_{1} X^{4}+3 \Pi_{2} \Pi_{3} X^{4}\left[1-\left(\frac{X}{J}\right)^{2 / 3}\right]=0, \quad X<J,
$$

where

$$
\begin{aligned}
& X \equiv k_{c} \eta=k_{c}\left(\frac{\nu^{3}}{\epsilon}\right)^{1 / 4}, \quad \Pi_{1} \equiv \frac{2 \widetilde{S}_{i j} \widetilde{S}_{i j}}{\left(k_{c}^{4} \nu^{2}\right)} \\
& \Pi_{2} \equiv \frac{\mathcal{K}_{0} \epsilon^{2 / 3}}{k_{c}^{8 / 3} \nu^{2}}, \quad \Pi_{3} \equiv \frac{\widetilde{S}_{i j}\left(\delta_{i j}-e_{i}^{v} e_{j}^{v}\right)}{\left(2 k_{c}^{2} \nu\right)} .
\end{aligned}
$$

Note that the group $\mathcal{K}_{0} \epsilon^{2 / 3}$ is preserved in $\Pi_{2}$. The parameter $\Pi_{1}$ is essentially twice the square of the grid-scale Reynolds number. It follows from (10) and (25) that $\Pi_{3}<0$ corresponds to transfer from resolved to subgrid scales (cascade) while $\Pi_{3}>0$ corresponds to transfer to resolved scales (backscatter). Given $\mathcal{K}_{0} \epsilon^{2 / 3}$ from (20), then (24) can be solved for $X$ by Newton's method at each gridpoint, and it is not necessary to solve a coupled system for either the whole domain or along homogeneous flow directions. The subgrid-kinetic energy and therefore the SGS tensor can then be computed for $X<J$. When $X>J$, the flow is estimated to be locally resolved $\left(k_{c}>J \eta^{-1}\right)$ and the SGS tensor is set to zero. We remark that this is a smooth transition at $X=J$ with no discontinuity in $\tau_{i j}$. A brief analysis of (24) is given in Appendix C. 


\section{NUMERICAL METHODS AND MODEL IMPLEMENTATION}

\section{A. Decaying isotropic turbulence}

The standard numerical method for the simulation of incompressible, turbulent flow in a cubic box with periodic boundary conditions is a pseudo-spectral Fourier-Galerkin technique. ${ }^{25}$ The physical-space version of the model was implemented in the pseudo-spectral code used by Misra and Pullin. ${ }^{19}$ In addition, decaying isotropic turbulence has also been simulated using compact finite differences. ${ }^{26}$ This method, though less efficient than the spectral method, was used to test the new version of the model with a numerical method operating purely in physical space. The compact finite difference code employs the internal iterations technique. ${ }^{27}$ This method uses operator splitting for the time advancement of the Navier-Stokes equations, but the pressure correction step is performed iteratively. Otherwise, using a standard pressure correction step with compact finite difference discretization would result in a rather complicated discrete Poisson operator. Instead, a simplified Poisson operator is used, and the resulting error is corrected iteratively. The iteration variable is a correction to the pressure which vanishes if the velocity field satisfies the divergence-free condition. Therefore the residual of the iteration is a direct indicator of the remaining divergence defect in the velocity field. In the present implementation, fourth-order compact finite differences are used in all three space dimensions on a fully staggered grid.

The pseudo-spectral method is fully de-aliased by the "3/2"'-technique. No de-aliasing has been applied in the compact finite difference method. Tests with the compact finite difference solver for the decaying turbulence case have been performed both with the divergence form and the skewsymmetric form of the nonlinear term, but results obtained with the divergence form were of the same or better quality than those obtained with the skewsymmetric form (only results for the divergence form will be shown).

\section{B. Channel flow}

The code for simulating the flow in a channel (periodic boundary conditions in streamwise and spanwise direction) uses a hybrid polynomial/Fourier-Galerkin method. ${ }^{28}$ Fourier expansions are used in the two homogeneous directions. The discretization in the wall-normal direction uses Lagrangian interpolation polynomials on the Gauss-LobattoLegendre (GLL) points. ${ }^{25}$ In the coordinate system used for the channel flow simulation, the streamwise direction is the $x$ - or one-direction, the spanwise direction is the $y$ - or twodirection and the wall-normal direction is the $z$ - or threedirection. The code can be run using either constant mean pressure gradient or constant flowrate to drive the channel flow. In every case, the simulations were run until a statistically steady state was reached as verified by symmetry in the obtained mean and rms velocity profiles, and by the magnitude of the wall-shear stresses. Then the simulation was run further to collect the statistics for the results shown here.

The skewsymmetric form of the non-linear terms is used, but tests have also been performed with additional dealiasing methods added to the code: For the homogeneous directions, the standard " $2 / 3$ " technique has been applied. (This method has higher computational overhead, but was easier to implement for the parallel version of the code than the " $3 / 2$ " technique.)

For the polynomial discretization, an analogous dealiasing method has been devised and applied: Since the velocity field is known on the GLL points, its discrete Legendre-polynomial coefficients can be computed. Those coefficients are then truncated. That is, if polynomials up to order $N$ are used in the discretization, coefficients of order greater than $M$ (with $M / N \leqslant 1$ ) are set to zero. Then the inverse polynomial transform is performed to recover the values of the field on the GLL points. This process, by which the de-aliased values $f_{d a}\left(z_{j}\right)$ of a discrete function $f\left(z_{j}\right)$, which is given at the GLL points $z_{j}$, are obtained, is described by the equation

$$
f_{d a}\left(z_{j}\right)=\sum_{k=0}^{M}\left(\frac{1}{\gamma_{k}} \sum_{n=0}^{N} f\left(z_{n}\right) L_{k}\left(z_{n}\right) w_{n}\right) L_{k}\left(z_{j}\right),
$$

where $L_{k}$ is the $k$ th order Legendre polynomial, $\gamma_{i}$ are known normalization factors, and $w_{i}$ are the GLL weights, which are also known. For the present work, $M / N=2 / 3$ was used.

\section{Model implementation}

The model implementation is similar for all numerical methods used, both for decaying isotropic turbulence and channel flow. As input, the model needs the local $\widetilde{U}_{i}$ and $\partial \widetilde{U}_{i} / \partial x_{j}$. The first step is to compute $\widetilde{F}_{2}^{c}$, at each grid point. This is done by approximating the circular average by an average over the four neighboring grid points in the plane. To account for nonuniform grids, we invoke Kolmogorov's inertial-range form ${ }^{29} F_{2} \sim(\epsilon r)^{2 / 3}$, which also follows from (9) and (15) without additional assumptions. This method was also used by Lesieur and Métais. ${ }^{1}$ For all present LES the circular average is performed in a plane normal to the three-direction (wall-normal for channel flow), and we obtain

$$
\begin{aligned}
\widetilde{F}_{2}^{c}= & \frac{1}{4} \sum_{i=1,2}\left[\left\|\widetilde{\mathbf{U}}(\mathbf{x})-\widetilde{\mathbf{U}}\left(\mathbf{x}+\Delta x_{i}^{+} \mathbf{e}_{i}\right)\right\|^{2}\left(\frac{r}{\Delta x_{i}^{+}}\right)^{2 / 3}\right. \\
& \left.+\left\|\widetilde{\mathbf{U}}(\mathbf{x})-\widetilde{\mathbf{U}}\left(\mathbf{x}-\Delta x_{i}^{-} \mathbf{e}_{i}\right)\right\|^{2}\left(\frac{r}{\Delta x_{i}^{-}}\right)^{2 / 3}\right],
\end{aligned}
$$

where $\Delta x_{i}^{+}$and $\Delta x_{i}^{-}$are the grid spacing in positive and negative $i$-direction, respectively (for the numerical methods used in the present work, $\Delta x_{i}^{+}=\Delta x_{i}^{-}$for $\left.i=1,2\right)$. The separation length for the structure function is set to $r$ $=\sqrt{\Delta x_{1} \Delta x_{2}}$, i.e., the geometric mean of the grid spacings in the $x_{1}-x_{2}$ plane where the structure function is computed.

Next, the orientation of the subgrid-vortex structures is determined. We assume alignment with eigenvectors of the resolved rate-of-strain tensor $\widetilde{S}_{i j}=\left(\partial \widetilde{U}_{i} / \partial x_{j}+\partial \widetilde{U}_{j} / \partial x_{i}\right) / 2$. Given the eigenvectors $\tilde{\mathbf{e}}_{1}, \tilde{\mathbf{e}}_{2}$, and $\tilde{\mathbf{e}}_{3}$ of $\widetilde{S}_{i j}$, with corresponding eigenvalues $\lambda_{1}<\lambda_{2}<\lambda_{3}$, we will use versions of 

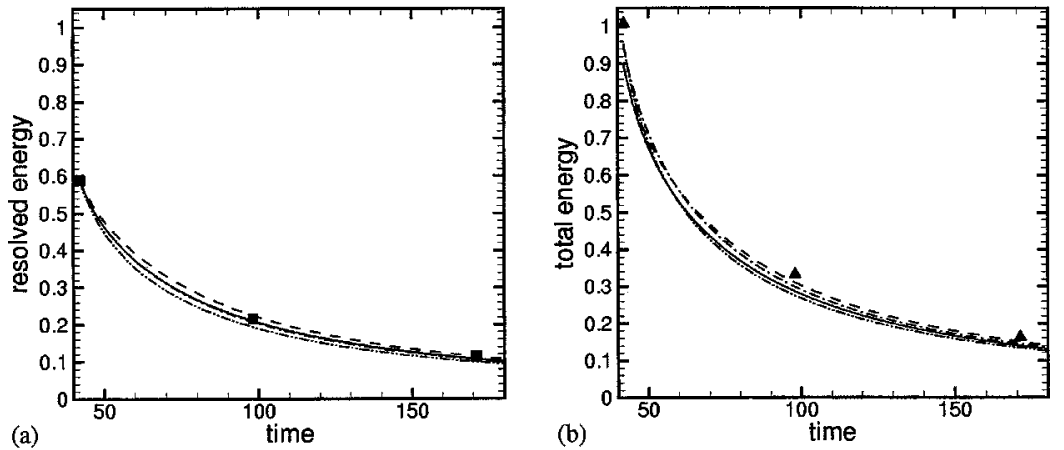

FIG. 2. Decay of (a) resolved-scale and (b) total kinetic energy, Fourier-Galerkin code, $---: \tilde{\mathbf{e}}_{2}+\widetilde{\mathbf{e}}_{3}$ model with $J=1 ;-: \tilde{\mathbf{e}}_{3}$ with $J=1 ;-\cdots: \tilde{\mathbf{e}}_{2}+\tilde{\mathbf{e}}_{3}$ with $J \rightarrow \infty ; \cdots$ : $\tilde{\mathbf{e}}_{3}$ with $J \rightarrow \infty$; symbols: data of Comte-Bellot and Corrsin (Ref. 30) filtered for (a).

the model, where the subgrid structures are assumed to be aligned with $\tilde{\mathbf{e}}_{3}$ only $\left(\tilde{\mathbf{e}}_{3}\right.$ model), or where a fraction $\lambda$ $\equiv \lambda_{3} /\left(\left|\lambda_{2}\right|+\lambda_{3}\right)$ of the structures is aligned with $\widetilde{\mathbf{e}}_{3}$, and the remainder $(1-\lambda)$ with $\tilde{\mathbf{e}}_{2}\left(\tilde{\mathbf{e}}_{2}+\tilde{\mathbf{e}}_{3}\right.$ model $)$. These versions, which use alignment with eigenvectors of $\widetilde{S}_{i j}$ only, do not model backscatter. In Ref. 19, other versions which include backscatter, e.g., using partial alignment with the resolvedscale vorticity vector, have been tested. But no significant improvements in the results of the simulations presented in the following would be expected by using different alignments.

Equation (20) can now be evaluated using (27). The integral in the denominator of (20) depends on $\psi$ and $r / \Delta$. Presently an analytical approximation to the integral was used (see Appendix B). An alternative is to use a twodimensional table lookup. The angle $\psi$ is known from the subgrid-vortex orientation. The ratio $r / \Delta$ is the ratio of the separation length $r$ used for the structure function and the cutoff length scale $\Delta\left(k_{c}=\pi / \Delta\right)$. In the present work $\Delta$ $=\left(\Delta z^{+}+\Delta z^{-}\right) / 2$ is used, i.e., the average of the distances to the two neighboring points in positive and negative $z$ direction.

When $J \rightarrow \infty$, the SGS kinetic energy can then be obtained immediately from (21). For finite $J$, (24) needs to be solved. This can be done efficiently with a Newton solver, for which an initial value is obtained by an analytical approximation; see Appendix $\mathrm{C}$ for details. When $X$ is known $K$ can be obtained from (22) and other quantities including $\mathcal{K}_{0}$ can be determined. The SGS stress tensor $\tau_{i j}$ then follows from (8).

\section{RESULTS AND DISCUSSION}

\section{A. Decaying isotropic turbulence}

The simulations of decaying isotropic turbulence were initialized to reproduce the experiments by Comte-Bellot and Corrsin. ${ }^{30}$ An initial, divergence-free velocity field was created in Fourier space such that its shell-summed energy spectrum matches the (truncated) spectrum given in Ref. 30 for $U_{\infty} t / M_{g}=42$. The phases of these initial Fourier coefficients are randomly distributed in the interval $[0,2 \pi]$. The time is given in the same nondimensionalization as used in Ref. 30, where $U_{\infty}=10 \mathrm{~m} / \mathrm{s}$ was the freestream air speed, and $M_{g}$ $=5.08 \mathrm{~cm}$ was the mesh width of the grid. The simulation uses internally the scaling $U_{\text {ref }}=\sqrt{3 U_{0}^{\prime 2} / 2}, L_{\mathrm{ref}}=L /(2 \pi)$, and $t_{\text {ref }}=L_{\text {ref }} / U_{\text {ref }}$, where $\sqrt{U_{0}^{\prime 2}}=0.222 \mathrm{~m} / \mathrm{s}$ is the magnitude of the velocity fluctuations at $U_{\infty} t / M_{g}=42$, and $L$ $=11 M_{g}$ is the size of the computational box. The results of the simulations are given using this velocity scale, but the time scale $M_{g} / U_{\infty}$.

First, we discuss the results obtained using the pseudospectral Galerkin code. All simulations used a resolution of $32^{3}$ Fourier modes. Simulations have been performed with $J \rightarrow \infty$ and $J=1$. The decay of the kinetic energy (per unit mass) is shown in Fig. 2. The resolved-scale contribution of the energy is computed directly from the resolved-scale velocity field, and is shown in Fig. 2(a). For comparison, the energy spectra given in Ref. 30 have been truncated to the resolution of the simulation, so that the experimental data can be decomposed in resolved-scale and subgrid-scale energy. The filtered experimental data for the resolved scales are shown as symbols in Fig. 2(a). From the SGS model, we also obtain an estimate for the kinetic energy contained in the subgrid scales. This is added to the resolved-scale energy to obtain an estimate for the total kinetic energy, which can be compared directly to the unfiltered experimental data, see Fig. 2(b). At our resolution of $32^{3}$, some $30 \%$ of the total subgrid energy is contained in the subgrid components midway through the simulation.

The comparison of the energy decay shows that the $\tilde{\mathbf{e}}_{2}$ $+\widetilde{\mathbf{e}}_{3}$ model is slightly less dissipative than the $\widetilde{\mathbf{e}}_{3}$ model, which can be explained by the fact that the eigenvector $\widetilde{\mathbf{e}}_{3}$ corresponds to the direction of maximum stretch. It can also be seen that the $J=1$ version is less dissipative than the $J$ $\rightarrow \infty$ version. The results for the decay of the total energy are somewhat too low, especially at the early stages of the simulation, which indicates that the model slightly underestimates the subgrid-kinetic energy. The discrepancies at early simulation times are likely to be attributable to the initial conditions with random phases. At the beginning of each simulation, the high-order correlations first need to develop, causing an initial transient period. But taking into account that this is an estimate for the total kinetic energy, the largeeddy simulations with the physical-space version of the stretched-vortex model can simulate the decay in satisfactory agreement with the experimental data.

The shell-summed, three-dimensional energy spectra, including the modeled subgrid spectra, are shown in Fig. 3. The model estimate of the factor $\mathcal{K}_{0} \epsilon^{2 / 3}$ determines the vertical offset in the subgrid spectrum. This estimate from the 

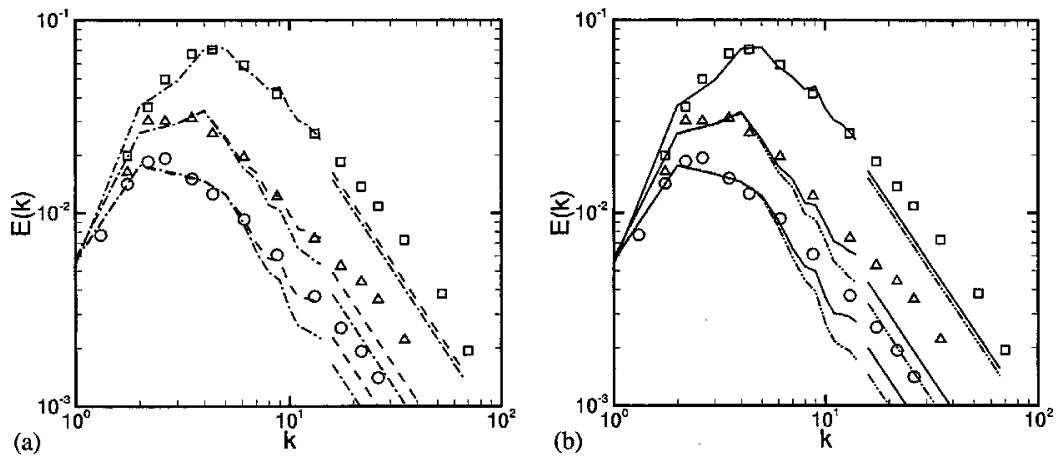

FIG. 3. Energy spectra for initial and two subsequent times (including the modeled subgrid spectra) (a) $\tilde{\mathbf{e}}_{2}$ $+\tilde{\mathbf{e}}_{3}$ model, (b) $\tilde{\mathbf{e}}_{3}$ model; line patterns as in Fig. 2, symbols: data from Ref. 30.

physical-space version satisfies the continuity requirement between the resolved and subgrid parts of the spectra to a satisfactory degree without enforcing this condition explicitly as in earlier versions of the model. ${ }^{19}$ Compared to the experimental data, the subgrid spectra are somewhat low, in accordance with the trend observed in the results for the total kinetic energy, but acceptable as a model estimate. The results for the resolved-scale spectrum show some differences depending on the choice of $J$. The result for the $\tilde{\mathbf{e}}_{2}+\tilde{\mathbf{e}}_{3}$ model with $J=1$ shows satisfactory agreement with the experimental data, while the spectra for the $J \rightarrow \infty$ version show that too much energy is removed from the highest resolved wave number modes in this version of the model. Since these differences occur at higher wave numbers they do not have a strong influence on the decay of the kinetic energy. The results also show that the model is only weakly dependent on the choice of alignment for the subgrid-vortex structure $\left(\tilde{\mathbf{e}}_{2}\right.$ $+\tilde{\mathbf{e}}_{3}$ model versus $\tilde{\mathbf{e}}_{3}$ model).

The energy decay from simulations with the compact finite difference code is shown in Fig. 4. The resolution for these simulations was $32^{3}$ gridpoints. According to these results, the model also performs satisfactorily within a pure physical-space numerical method. The results for the decay of the resolved-scale energy are of comparable quality as those obtained with the spectral method. The total kinetic energy also shows similar trends: The model estimate for the subgrid-kinetic energy is somewhat too low, especially for early times. But this simulation was also able to give a satisfactory estimate for the decay of the total kinetic energy.

\section{B. Channel flow}

Simulations for the channel flow geometry described in Sec. III B have been performed for three different Reynolds numbers. In the first case, the Reynolds number based on the wall shear velocity was $\operatorname{Re}_{\tau}=u_{\tau} \delta / \nu=180$. The wall shear velocity is defined as $u_{\tau} \equiv\left(\tau_{W} / \rho\right)^{1 / 2}$, where $\tau_{W}$ is the shear stress at the wall, and $\rho$ is the density. $\nu$ is the kinematic viscosity and $\delta$ is the channel half-width. This $\operatorname{Re}_{\tau}$ corresponds to $\operatorname{Re}_{c} \approx 3300$, where $\operatorname{Re}_{c}$ is the Reynolds number based on mean centerline velocity (and channel half-width). For the second case, the nominal Reynolds number was $\operatorname{Re}_{\tau}=590\left(\operatorname{Re}_{c} \approx 12500\right)$, and in the last case, it was $\operatorname{Re}_{\tau}$ $=1017\left(\operatorname{Re}_{c} \approx 22800\right)$.

The parameters for the different simulations performed are shown in Table I. The column "LES" indicates whether the model has been turned on $(Y)$ or off $(N)$ for that particular case. (Runs without model, i.e., under resolved simulations, have been performed for comparison.) We also compare cases with and without the de-aliasing described in Sec. III B. The resolution, i.e. the number of gridpoints, is given in a way that accounts for the de-aliasing. We call this the "effective" resolution. If no de-aliasing is used, the numbers given in the table are simply the number of modes used in the simulation. When the de-aliasing (using the " $2 / 3$ " method) is turned on, however, the number of modes used in the simulation is $50 \%$ higher than the numbers given here. But the upper $1 / 3$ of the modes in the Fourier and polynomial spectra are set to zero for de-aliasing. The number of modes
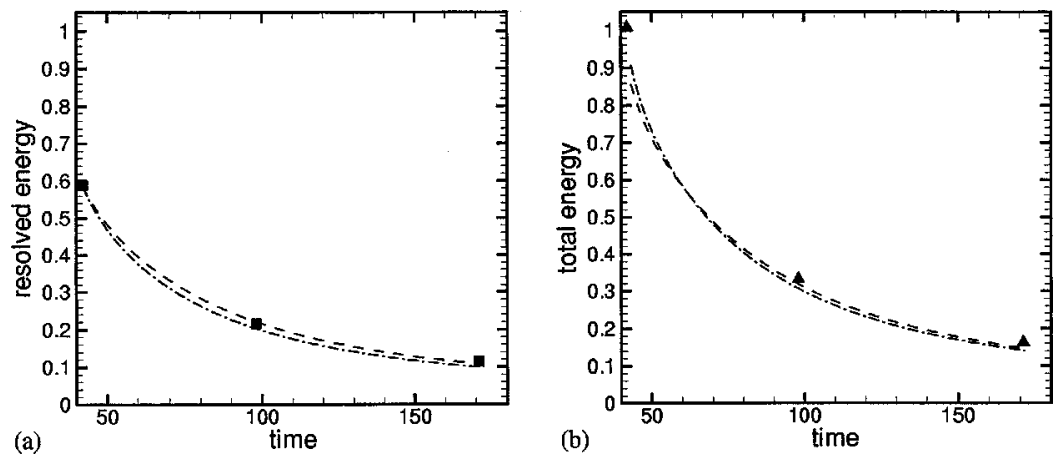

FIG. 4. Decay of (a) resolved-scale and (b) total kinetic energy, compact finite difference code, ---: $\tilde{\mathbf{e}}_{2}+\tilde{\mathbf{e}}_{3}$ model with $J=1 ;-\cdots:$ : $\tilde{\mathbf{e}}_{2}+\tilde{\mathbf{e}}_{3}$ model with $J \rightarrow \infty$. 
TABLE I. Important parameters for the channel flow simulations

\begin{tabular}{|c|c|c|c|c|c|c|c|c|c|c|}
\hline \multirow[b]{2}{*}{ Case } & \multirow[b]{2}{*}{$\operatorname{Re}_{\tau}$} & \multicolumn{2}{|c|}{ Domain size } & \multirow[b]{2}{*}{ LES } & \multirow[b]{2}{*}{ De-aliasing } & \multirow{2}{*}{$\begin{array}{l}\text { Effective Res. } \\
N_{x}^{\prime} \times N_{y}^{\prime} \times N_{z}^{\prime}\end{array}$} & \multicolumn{4}{|c|}{ Grid spacing } \\
\hline & & $L_{x} / \delta$ & $L_{y} / \delta$ & & & & $\Delta x^{+}$ & $\Delta y^{+}$ & $\Delta z_{\min }^{+}$ & $\Delta z_{\max }^{+}$ \\
\hline 1 & 180 & $4 \pi$ & $2 \pi$ & $Y$ & $Y$ & $32 \times 32 \times 43$ & 71 & 35 & 0.73 & 13.3 \\
\hline 2 & 180 & $4 \pi$ & $2 \pi$ & $Y$ & $N$ & $32 \times 32 \times 65$ & 71 & 35 & 0.32 & 8.76 \\
\hline 3 & 180 & $4 \pi$ & $2 \pi$ & $N$ & $Y$ & $32 \times 32 \times 43$ & 71 & 35 & 0.73 & 13.3 \\
\hline 4 & 1017 & $\frac{5}{2} \pi$ & $\pi$ & $Y$ & $Y$ & $48 \times 64 \times 65$ & 166 & 50 & 1.79 & 49.5 \\
\hline 5 & 1017 & $\frac{5}{2} \pi$ & $\pi$ & $Y$ & $N$ & $48 \times 64 \times 65$ & 166 & 50 & 1.79 & 49.5 \\
\hline 6 & 1017 & $\frac{5}{2} \pi$ & $\pi$ & $N$ & $N$ & $48 \times 64 \times 65$ & 166 & 50 & 1.79 & 49.5 \\
\hline $7^{\mathrm{a}}$ & 180 & $4 \pi$ & $2 \pi$ & $\mathrm{Y}$ & $Y$ & $32 \times 32 \times 43$ & 71 & 35 & 0.73 & 13.3 \\
\hline $8^{\mathrm{b}}$ & 1017 & $\frac{5}{2} \pi$ & $\pi$ & $Y$ & $N$ & $48 \times 64 \times 65$ & 166 & 50 & 1.79 & 49.5 \\
\hline $9^{b, c}$ & 590 & $2 \pi$ & $\pi$ & $Y$ & $N$ & $64 \times 64 \times 97$ & 58 & 29 & 0.46 & 19.2 \\
\hline $10^{\mathrm{b}, \mathrm{c}}$ & 590 & $2 \pi$ & $\pi$ & $N$ & $N$ & $64 \times 64 \times 97$ & 58 & 29 & 0.46 & 19.2 \\
\hline
\end{tabular}

${ }^{\mathrm{a}}$ Case 7 is a realization of the same flow as case 1 at a different time.

${ }^{\mathrm{b}}$ Uses the $\tilde{\mathbf{e}}_{2}+\tilde{\mathbf{e}}_{3}$ model version.

${ }^{\mathrm{c}}$ Run with constant flowrate.

which are retained after de-aliasing is given in the table as "effective" resolution.

The simulations have been carried out using Eqs. (1) and (2) in nondimensionalized form with $u_{\tau}$ as velocity scale and $\delta$ as length scale. The time scale is then $\delta / u_{\tau}$. Quantities scaled by wall variables are denoted by the superscript + . For the presentation of the results, the wall-normal coordinate is also given in wall coordinates: $z^{+} \equiv z u_{\tau} / \nu$. The statistics have been computed using plane- and time-averaging. The $\operatorname{Re}_{\tau}=180$ and $\operatorname{Re}_{\tau}=1017$ cases were performed using a constant mean pressure gradient to drive the flow. In this case, because of the global momentum balance, the (mean) shear stress at the wall will always go to the nominal value as determined by $\operatorname{Re}_{\tau}$ when the flow reaches steady state. For the present simulations (cases 1-8), the mean value was within $1 \%$ to $2 \%$ of the nominal value, and the fluctuations typically were less than $10 \%$ of the mean. For the $\operatorname{Re}_{\tau}$ $=590$ case , the constant flowrate version of the code was used. (The flowrate was set to the value computed from the mean velocity profile of the DNS. ${ }^{31}$ ) This method of driving the flow is known to significantly reduce the time required to reach steady state. In this case, the resulting value of the wall shear stress depends on the variable pressure gradient which is adjusted dynamically to maintain constant flowrate. Most of the channel flow simulations were performed with the $\tilde{\mathbf{e}}_{3}$ model version, except where otherwise noted in Table I. For the $\operatorname{Re}_{\tau}=1017$ case, results for both the $\tilde{\mathbf{e}}_{3}$ and the $\tilde{\mathbf{e}}_{2}+\tilde{\mathbf{e}}_{3}$ version (case 8 ) were available. It was found that the results of case 8 for the mean and rms quantities (not shown) are very close to the results obtained for alignment with $\tilde{\mathbf{e}}_{3}$ only. The same run (case 8) was used to collect additional statistics for the turbulence-energy balances, which will be shown here. All channel flow large-eddy simulations presented here used the $J \rightarrow \infty$ version of the model, which is less complicated and computationally less expensive than a version with
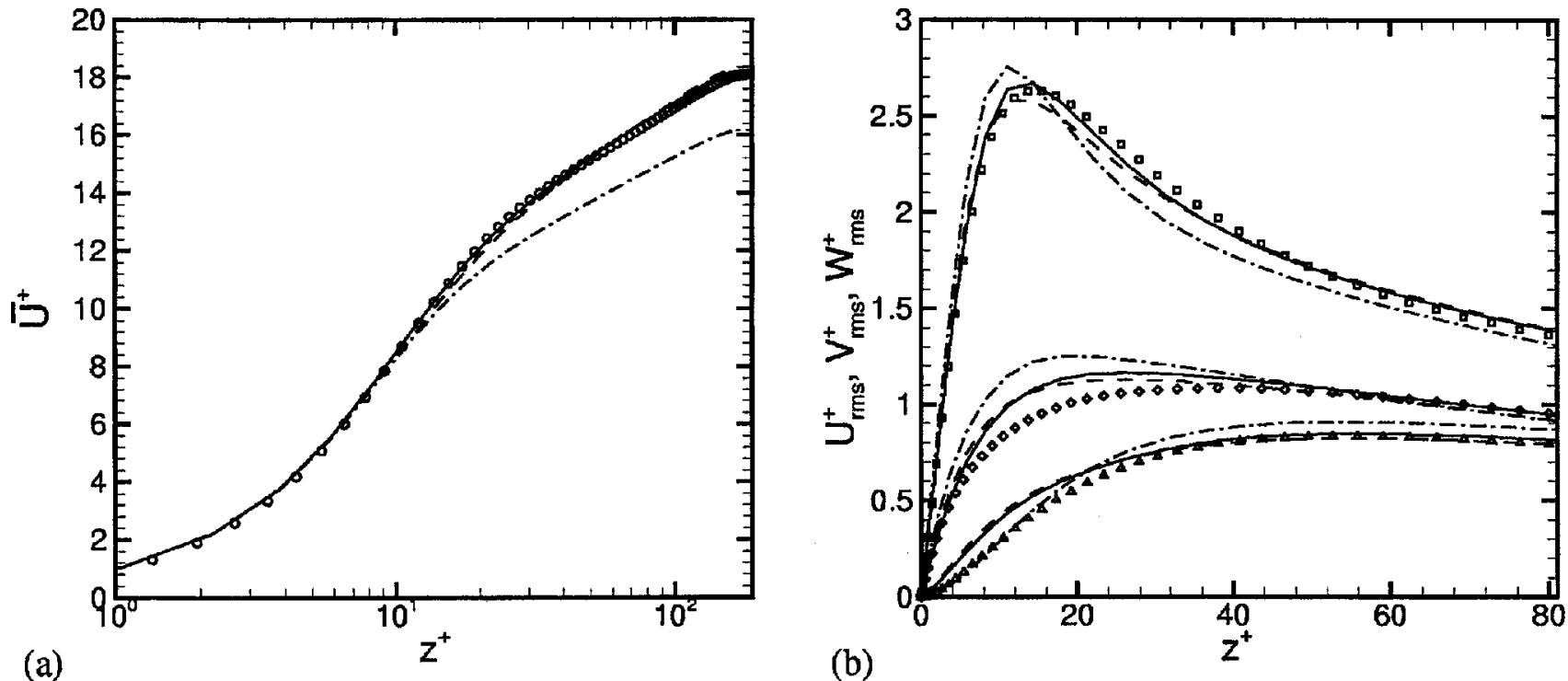

FIG. 5. Channel flow at $\operatorname{Re}_{\tau}=180$ (a) mean velocity and (b) root-mean-square velocity fluctuations (resolved plus subgrid). —: LES, with de-aliasing (case 1); ---: LES, no de-aliasing (case 2); - . -: no model, with de-aliasing (case 3); symbols: data (unfiltered) from DNS by Kim, Moin, and Moser (Ref. 32) $\bigcirc$ : $\bar{U}^{+} ; \square: U_{\mathrm{rms}}^{+} ; \diamond: V_{\mathrm{rms}}^{+} ; \triangle: W_{\mathrm{rms}}^{+}$. 

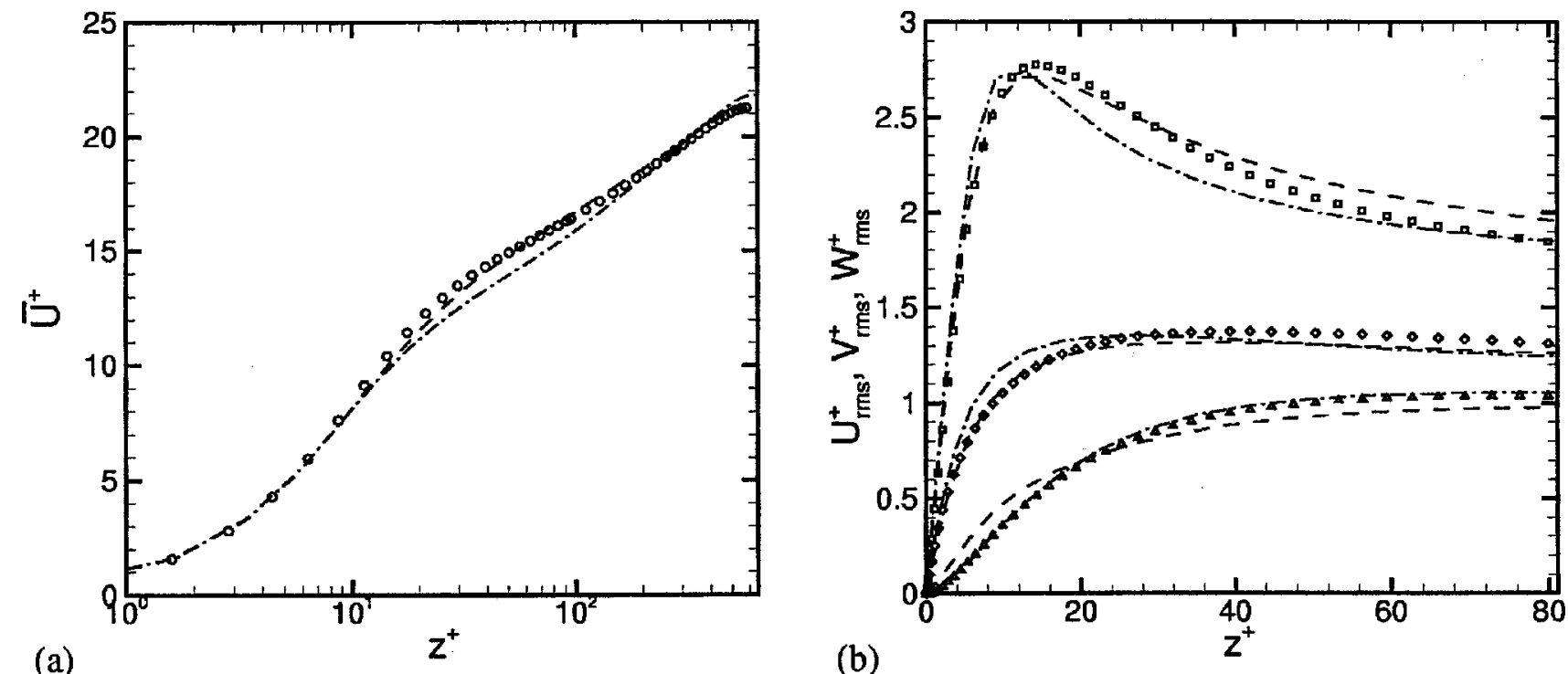

FIG. 6. Channel flow at $\operatorname{Re}_{\tau}=590$, simulation with constant flowrate (a) mean velocity and (b) root-mean-square velocity fluctuations (resolved plus subgrid). - - -: LES, no de-aliasing (case 9); - - -: no model, no de-aliasing (case 10); symbols: data (unfiltered) from DNS by Moser, Kim, and Mansour (Ref. 31), $\bigcirc$ : $\bar{U}^{+}$(only every fourth point shown, except close to the wall every second point); $\square: U_{\mathrm{rms}}^{+} ; \diamond: V_{\mathrm{rms}}^{+} ; \triangle: W_{\mathrm{rms}}^{+}$. Note that in (a) the LES result is covered by the symbols near the centerline.

finite $J$. The results of the decaying isotropic turbulence test indicate that the simpler $J \rightarrow \infty$ version performs sufficiently well for this application. This may not necessarily be true for all cases, especially when Reynolds-number effects become significant.

When computing the rms velocity fluctuations, it is necessary to distinguish between two different decompositions which are used in this context: On one hand, a flow quantity $U$ can be subjected to the LES decomposition in resolved scale contribution $\widetilde{U}$ and subgrid-scale component $u$. On the other hand, Reynolds averaging can be performed to decompose $U$ in a mean quantity $\bar{U}$ and the fluctuating component $U^{\prime}$. The overbar denotes an ensemble average (which will in praxis be computed using spatial and temporal averages). Combining these two decompositions we can write

$$
U=\overline{\tilde{U}}+\widetilde{U}^{\prime}+u^{\prime},
$$

and we assume $\overline{\tilde{U}}=\bar{U}$ and $u^{\prime}=u$.

Now consider the correlation tensor of the turbulent velocity fluctuations $\overline{U_{i}^{\prime} U_{j}^{\prime}}=\overline{U_{i} U_{j}}-\bar{U}_{i} \bar{U}_{j}$. Using Eq. (28), we obtain

$$
\overline{U_{i}^{\prime} U_{j}^{\prime}}=\overline{\widetilde{U}_{i}^{\prime} \widetilde{U}_{j}^{\prime}}+\overline{\widetilde{U}_{i}^{\prime} u_{j}^{\prime}}+\overline{\widetilde{U}_{j}^{\prime} u_{i}^{\prime}}+\overline{u_{i}^{\prime} u_{j}^{\prime}} .
$$

The first term is known, but the cross terms (correlation of resolved-scale, fluctuating velocities and subgrid-scale fluc-
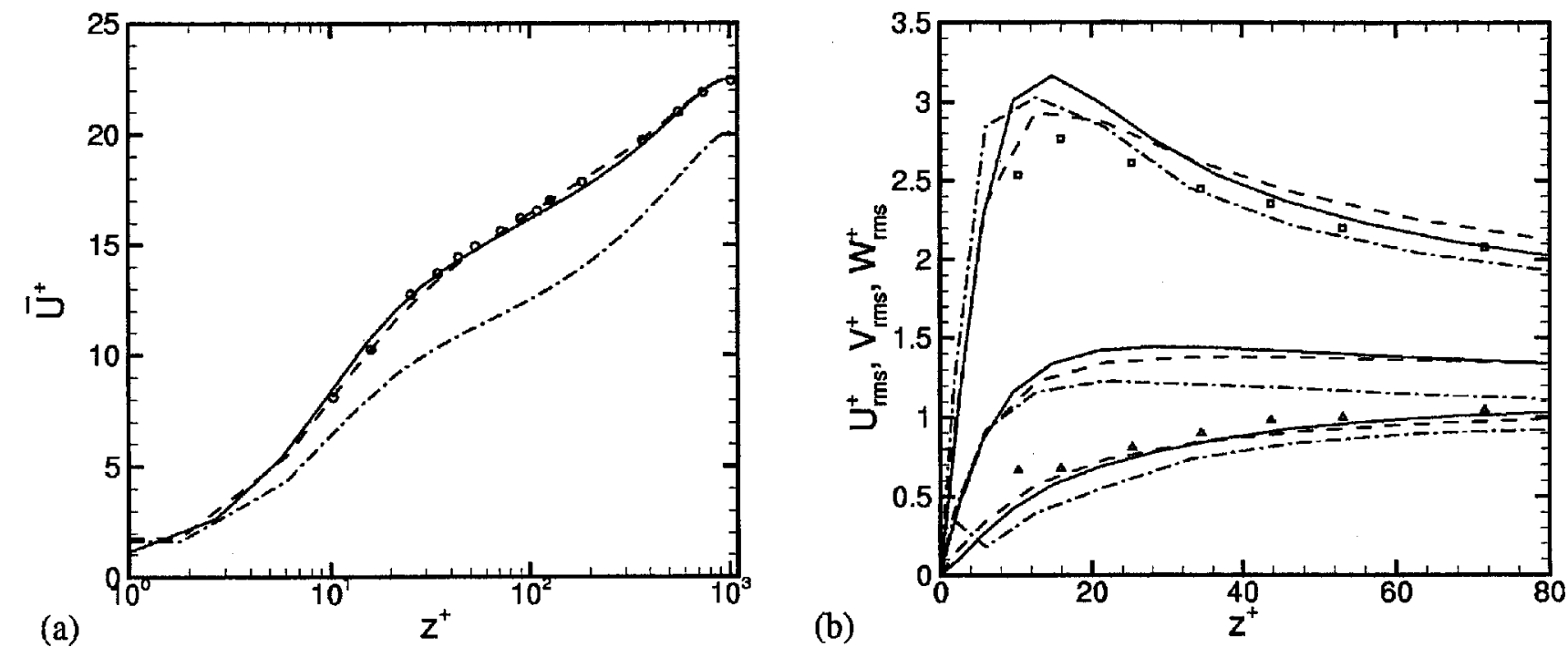

FIG. 7. Channel flow at $\operatorname{Re}_{\tau}=1017$ (a) mean velocity and (b) root-mean-square velocity fluctuations (resolved plus subgrid). — : LES, with de-aliasing (case 4); - - -: LES, no de-aliasing (case 5); - - -: no model, no de-aliasing (case 6); symbols: data (unfiltered) from experiments by Wei and Willmarth (Ref. 33) $\bigcirc: \bar{U}^{+} ; \square: U_{\text {rms }}^{+} ; \triangle: W_{\text {rms }}^{+}$. 
tuations) and the subgrid-scale correlation term are unknown in the context of LES. Only the last term is modeled. The best estimate for the rms quantities, which can be obtained from large-eddy simulations, is therefore

$$
\overline{U_{i}^{\prime} U_{j}^{\prime}} \approx \overline{\tilde{U}_{i}^{\prime} \tilde{U}_{j}^{\prime}}+\bar{\tau}_{i j} .
$$

We use this LES estimate for the rms velocity fluctuations (which requires knowledge of the full $\tau_{i j}$, not only the tracefree component of the tensor) and compare it with the (unfiltered) results from DNS and experiment. Thereby we avoid applying a filter defined only in the context of largeeddy simulations to DNS and experimental results. For experimental data, it may often not be possible at all to compute filtered rms velocities because the data necessary to perform the filtering operation may not be available.

\section{Mean and rms velocities for $\operatorname{Re}_{\tau}=180$}

Results for the $\operatorname{Re}_{\tau}=180$ flow are shown in Fig. 5. The time averaging for these results was performed over a simulation time of $\left(t_{\text {end }}-t_{\text {start }}\right) u_{\tau} / \delta=30$. The mean velocity [Fig. 5 (a)] of the de-aliased LES (case 1) shows good agreement with the DNS data by Kim, Moin, and Moser. ${ }^{32}$ Comparison with case 3 (no model) shows the significant contribution of the LES model. The results from the LES without de-aliasing (case 2) are little different from those with de-aliasing, although there is some improvement noticeable in the buffer region and near the center of the channel. The differences between case 1 (with de-aliasing) and case 2 (without dealiasing) are slightly larger in the root-mean-square velocity fluctuations [Fig. 5(b), shown for the near-wall region]. In particular, the streamwise fluctuations show some improvement when the de-aliasing is used. But both LES estimates, with and without de-aliasing, are somewhat too high for the spanwise fluctuations $V_{\text {rms }}$. As described above, these are LES estimates for the total velocity fluctuations, which are compared to the unfiltered results of DNS in Fig. 5(b).

\section{Mean and rms quantities for $\operatorname{Re}_{\tau}=590$}

The mean and rms velocity profiles for $\operatorname{Re}_{\tau}=590$ (Fig. 6) also show good results for the LES (case 9). We recall that for this $\operatorname{Re}_{\tau}$, constant flowrate was used in the simulation, and for both, cases 9 (LES) and 10 (no model), the same flowrate was prescribed. Therefore the influence of the model is less pronounced in the mean profile (though still clearly visible). A good indication of the quality of the re-

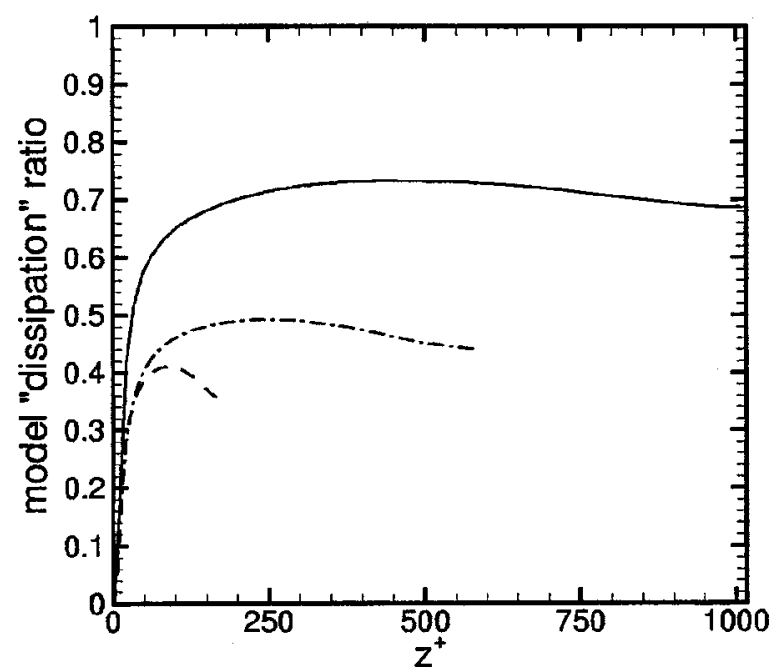

FIG. 8. Model 'dissipation', ratio $\varepsilon_{\mathrm{sgs}} /\left(\tilde{\epsilon}_{\mathrm{visc}}+\varepsilon_{\mathrm{sgs}}\right),-$ : $\operatorname{Re}_{\tau}=1017 ;-\cdot-$ : $\operatorname{Re}_{\tau}=590$, ---: $\operatorname{Re}_{\tau}=180$.

sults in this case is the actual $\mathrm{Re}_{\tau}$ as determined by the wall shear stress measured in the simulation results. We find $\operatorname{Re}_{\tau}=584$ for the LES (case 9), giving 1\% error compared to DNS, and $\operatorname{Re}_{\tau}=633$ (case 10) without the model. The time averaging for case 9 was performed over $\left(t_{\text {end }}-t_{\text {start }}\right) u_{\tau} / \delta$ $=9.75$, and for case 10 over $\left(t_{\text {end }}-t_{\text {start }}\right) u_{\tau} / \delta=8$. For this $\operatorname{Re}_{\tau}$, no de-aliased runs have been performed.

\section{Mean and rms quantities for $\mathrm{Re}_{\tau}=1017$}

The mean and rms velocity profiles for $\operatorname{Re}_{\tau}=1017$ are shown in Fig. 7. The time averaging for these results was performed over $\left(t_{\text {end }}-t_{\text {start }}\right) u_{\tau} / \delta=10$. Again the mean velocity profiles [Fig. 7(a)], both with and without de-aliasing, show good agreement with the reference data, in this case experimental data by Wei and Willmarth. ${ }^{33,34}$ The comparison with case 6 (no model, same grid resolution) shows the large influence of the model at this Reynolds number. The agreement of the LES estimates for the total rms velocities with the experimental data is not as good as for the mean velocities. In particular, the de-aliased result for the streamwise fluctuations overestimates the peak value and the error is larger than for the case without de-aliasing. The de-aliased result seems to show some improvement in predicting the wall-normal coordinate of the peak. Note that there are no experimental data for the spanwise fluctuations.
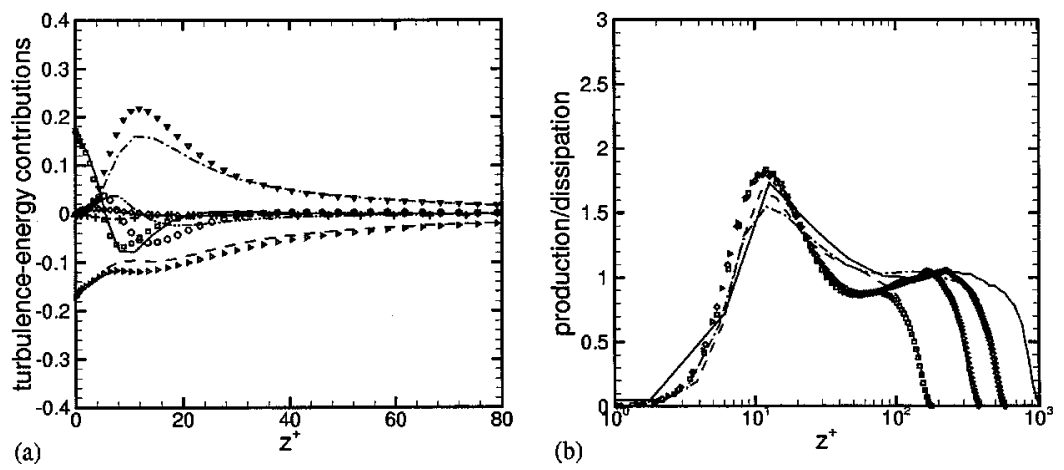

FIG. 9. Turbulence-energy equation (a) terms of resolved-scale balance (see text) for $\operatorname{Re}_{\tau}=180$ : -. -: production; ---: dissipation; - : surface work; - - --: convective diffusion of turbulence kinetic energy; --: convective diffusion of the internal energy (pressure term), + : sum of all terms. Also shown, DNS results (unfiltered) by Moser, Kim, and Mansour (Ref. 31), $\nabla$ : production; $\triangleright$ : dissipation; $\square$ : work of the tractions; $\bigcirc$ : diffusion of turbulence kinetic energy; $\diamond:$ diffusion of internal energy. (b) Ratio of production to dissipation (absolute value), - $\operatorname{Re}_{\tau}=1017 ;-\cdots: \operatorname{Re}_{\tau}=590,---: \operatorname{Re}_{\tau}=180$, symbols: DNS (Ref. 31), $\square: \operatorname{Re}_{\tau}=180 ; \triangleright: \operatorname{Re}_{\tau}$ $=395 ; \diamond: \operatorname{Re}_{\tau}=590$. 


\section{Model "dissipation"}

The behavior of the model, in particular in the region very near to the walls, and its influence on the resolved scales can be illustrated by plotting the ratio $\varepsilon_{\mathrm{sgs}} /\left(\varepsilon_{\mathrm{sgs}}\right.$ $+\tilde{\epsilon}_{\text {visc }}$ ) as a function of the wall-normal coordinate (Fig. 8). The quantity $\varepsilon_{\text {sgs }}$ was defined in Eq. (10), it has the effect of a model "dissipation" on the resolved scales. The resolvedscale, viscous dissipation is denoted by $\widetilde{\epsilon}_{\text {visc }} \equiv 2 \nu \widetilde{S}_{i j} \widetilde{S}_{i j}$. For the highest Reynolds number, the model contribution to the correct energy removal from the resolved scales is very significant, except for the region very close to the wall. For $z^{+} \rightarrow 0$, i.e., when approaching the wall, the model contribution rapidly decays to zero, due to the dynamic estimate of the factor $\mathcal{K}_{0} \epsilon^{2 / 3}$ using local circular-averaged structure functions. This vanishing model influence in the region very near to the wall, in connection with a reduced grid-spacing at least in the wall-normal direction, is typically required for large-eddy simulations of wall-bounded shear flows.

\section{Turbulence-energy balance}

In LES simulations, only the balance of the resolvedscale turbulence energy can be determined completely from the resolved quantities. For the channel flow, the following resolved-scale turbulence-energy equation is obtained after averaging over the homogeneous directions and time averaging:

$$
\begin{aligned}
& \frac{d}{d t} \overline{\left(\frac{1}{2} \tilde{U}_{j}^{\prime} \widetilde{U}_{j}^{\prime}\right)}=-\overline{\frac{\partial}{\partial x_{3}}\left[\tilde{U}_{3}^{\prime}\left(\frac{\tilde{P}^{\prime}}{\rho}+\frac{1}{2} \tilde{U}_{j}^{\prime} \tilde{U}_{j}^{\prime}\right)\right]}
\end{aligned}
$$

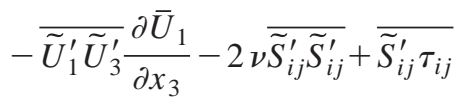

$$
\begin{aligned}
& +2 \nu \overline{\frac{\partial}{\partial x_{3}}\left(\tilde{U}_{i}^{\prime} \tilde{S}_{i 3}^{\prime}\right)}-\overline{\frac{\partial}{\partial x_{3}}\left(\widetilde{U}_{i}^{\prime} \tau_{i 3}\right)} .
\end{aligned}
$$

For steady state, the left-hand side vanishes. (Note that $d / d t$ denotes the material derivative, but the convective terms vanish for a steady channel flow.) Accordingly, the contributions on the right-hand side must be in balance. The first term on the right-hand side is the convective diffusion of the total turbulence energy, which consists of the internal energy (pressure term) and the kinetic energy, the second is the production term, the third is the viscous dissipation, the fourth term is the subgrid "dissipation," i.e., the energy transfer from the resolved scales to the subgrid scales, the fifth term is the surface work done by the viscous shear stresses of the turbulent motions, and the last term is the surface work done by the subgrid stresses. Those terms are shown for $\operatorname{Re}_{\tau}$ $=180$ (case 7) in Fig. 9(a) (the two dissipation terms are lumped together, as are the two surface work terms). In Fig. 9(b), the ratio of the production term to the dissipation term (absolute values) is plotted. Unfiltered results from the DNS by Moser, Kim, and Mansour ${ }^{31}$ are also shown in the figures. This allows to identify those terms in the resolved-scale energy balance where significant contributions are missing compared to the energy balance for the full flowfield. Par- ticularly large differences are visible in the production term and the convective-diffusion term of the kinetic energy.

For those two terms, additional contributions from interactions with the subgrid scales can be estimated using themodeled SGS tensor:

Production $\approx-\overline{\widetilde{U}_{1}^{\prime} \widetilde{U}_{3}^{\prime}} \frac{\partial \bar{U}_{1}}{\partial x_{3}}-\bar{\tau}_{13} \frac{\partial \bar{U}_{1}}{\partial x_{3}}$ and

Convective diffusion of kinetic energy

$$
\approx-\overline{\frac{\partial}{\partial x_{3}}\left[\tilde{U}_{3}^{\prime}\left(\frac{1}{2} \tilde{U}_{j}^{\prime} \tilde{U}_{j}^{\prime}+\frac{1}{2} \tau_{k k}\right)\right] .}
$$

These are not exact expressions for the full production and kinetic-energy diffusion terms because their decomposition in resolved and subgrid component has additional contributions, but those cannot be estimated with a model for the SGS tensor. Including the known additional contributions, the terms of the turbulence-energy balance are plotted again in Fig. 10 for all three Reynolds numbers investigated here. The production term with the subgrid contribution in the $\operatorname{Re}_{\tau}=180$ case now matches the DNS result better. Significant disagreement remains, however, for the diffusion of turbulence-kinetic energy. The LES estimate, even though it includes a subgrid contribution (the convective diffusion of the subgrid-kinetic energy by the resolved-scale velocity), looks qualitatively similar to the result for the resolvedscales only, which was obtained by Moin and Kim. ${ }^{35}$

To assess the quality of the numerical results, the sum of the terms which appear in the right-hand side of Eq. (31) has been computed (i.e., without the additional subgrid contributions which are included in Fig. 10). The value of the sum, which should go to zero for a sufficiently large number of statistical samples, is plotted with " +" symbols. In the low and medium Reynolds number cases, this condition is satisfied to acceptable accuracy but in the high Reynolds number case [Fig. 10(c)], errors are visible very close to the wall. These errors have to be attributed to the relatively poor nearwall resolution at $\operatorname{Re}_{\tau}=1017$.

In Fig. 11 the ratio of the production including the subgrid contribution to the absolute value of the dissipation is shown. The LES estimates for $\operatorname{Re}_{\tau}=180$ and for $\operatorname{Re}_{\tau}$ $=1017$ are too high in the near-wall region compared to the DNS data. For $\operatorname{Re}_{\tau}=590$, this overestimate near the wall is reduced significantly. For this case, a somewhat finer grid (see the grid spacing in wall units in Table I) has been chosen, and the improvements in these higher-order statistics can likely be attributed to this higher resolution. For both higher Reynolds number cases, it is interesting to note that in the region where an inertial sublayer may be expected the ratio of production to dissipation is approximately unity although the curve does not have an exactly horizontal plateau, and the values are slightly above unity. The DNS data for intermediate Reynolds numbers seems to approach approximately the same value, however. But given the expected inaccuracies in these medium and high Reynolds number LES, it cannot be conclusively determined if there is a region in turbulent channel flow where production and dissipation are balanced. 


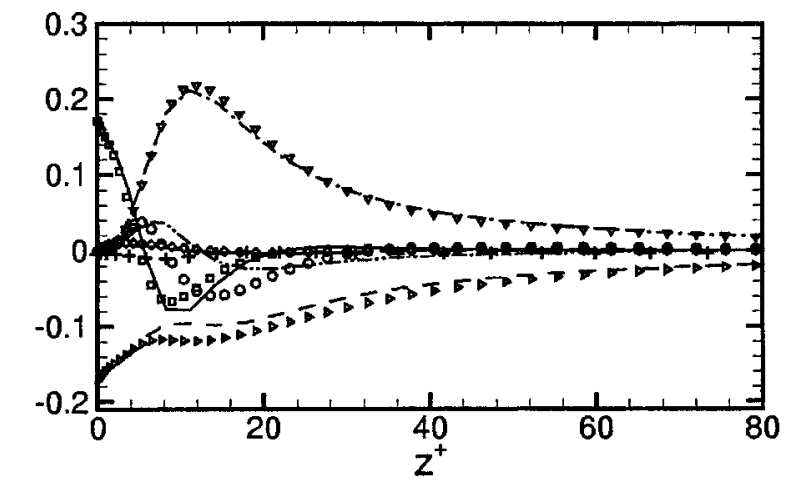

(a)

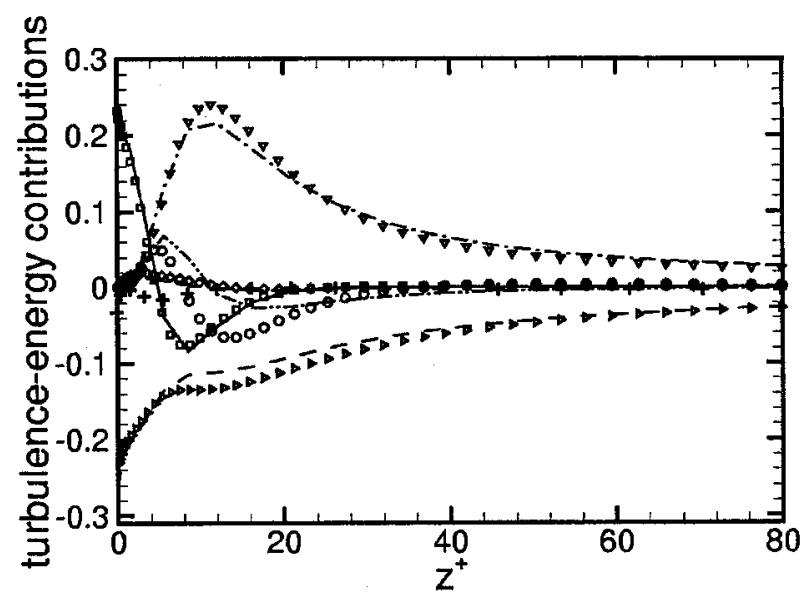

(b)

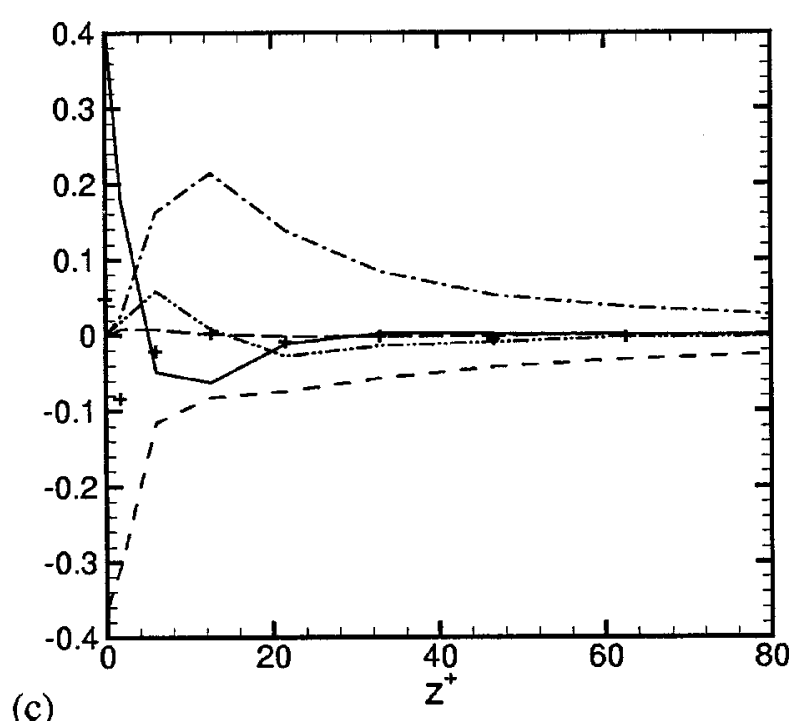

(c)

FIG. 10. Turbulence-energy balance including known additional subgrid terms (see text), (a) $\operatorname{Re}_{\tau}=180$ (case 7), (b) $\operatorname{Re}_{\tau}=590$ (case 9), (c) $\operatorname{Re}_{\tau}$ $=1017$ (case 8). ---: production with subgrid contribution; ---: dissipation; -: surface work; - - - - convective diffusion of turbulence kinetic energy with subgrid contribution; --: convective diffusion of the internal energy (pressure term), + : sum of the resolved-scale terms, i.e. right-hand side of Eq. (31). Symbols (DNS) as in Fig. 9(a): (a) $\operatorname{Re}_{\tau}=180$, (b) $\operatorname{Re}_{\tau}=590$.

\section{CONCLUDING REMARKS}

A physical-space version of the stretched-vortex subgrid-stress model has been proposed and tested. The

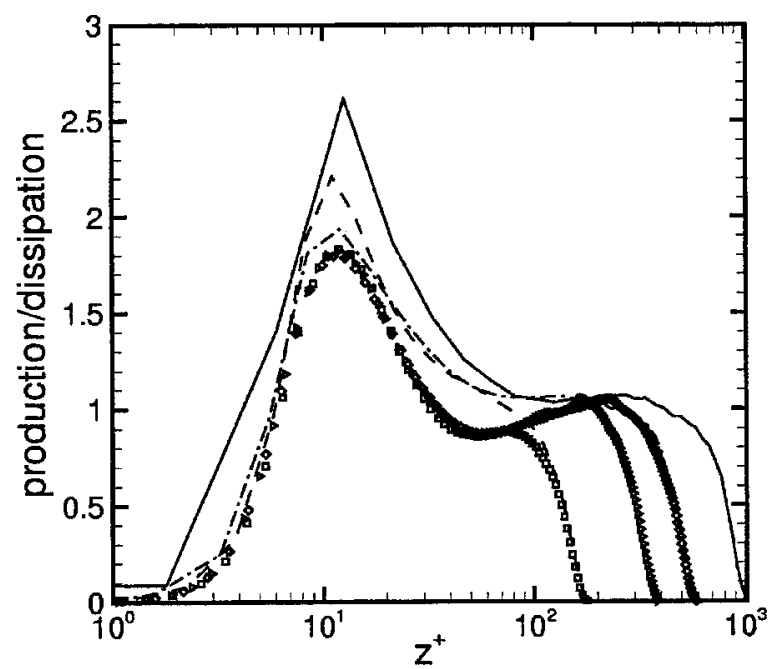

FIG. 11. Ratio of production (including known additional subgrid contribution) to dissipation (absolute value), - $: \operatorname{Re}_{\tau}=1017$ (case 8); - - : $\operatorname{Re}_{\tau}$ $=590$ (case 9), ---: $\operatorname{Re}_{\tau}=180$ (case 7), symbols: DNS (Ref. 31), $\square: \operatorname{Re}_{\tau}$ $=180 ; \triangleright: \operatorname{Re}_{\tau}=395 ; \diamond: \operatorname{Re}_{\tau}=590$.

stretched-vortex model is based on kinematic results for homogeneous anisotropic turbulence, which can be derived based on certain assumptions about the vorticity structure of subgrid scales. The physical-space version is intended to allow the implementation of the model in a localized manner while maintaining the desirable features of the stretchedvortex model, namely that no a priori parameters need to be adjusted for different flow geometries and a direct estimate of the subgrid-kinetic energy is provided. In the physicalspace version, the model is not restricted to geometries with homogeneous flow directions. We would therefore expect that it should be applicable to more complicated flow geometries than those considered presently. For complex geometries, the plane in which the circular average is performed should be chosen to be locally parallel to the wall. Using body-fitted coordinates, this can be expected to be relatively straightforward. Further away from the walls, the orientation of the circular-averaging plane should not have a strong influence on the model behavior. But actual tests will need to be carried out in order to confirm the applicability of the model in more general problems.

The model has been tested by comparison with DNS and experimental results for two different test cases, decaying box turbulence and turbulent channel flow. Mean flow profiles for channel flow at low and moderately high Reynolds numbers were predicted very well, and the agreement for rms velocity fluctuations was satisfactory. This may indicate that the model versions used here, which do not include backscatter, nevertheless worked well, at least as measured by commonly used quantities to describe such flows in the laboratory. The model appears to fulfill the principal purpose of an SGS model, namely to remove (on average) approximately the right amount of energy from the resolved scales.

Comparisons of higher-order statistics (turbulenceenergy budget) with DNS data showed some larger differences which may be indicative of the limitations of the model and also of the limits of large-eddy simulations of 
wall-bounded flows at high Reynolds numbers. To keep the computational costs to a reasonable amount, as is desired in large-eddy simulations, only relatively few grid points can be placed in the region very close to the wall.

\section{ACKNOWLEDGMENTS}

This work was supported in part by the National Science Foundation under Grant No. CTS-9634222. The channel flow simulations were greatly facilitated by the availability of the Cray T3E at the Pittsburgh Supercomputing Center (PSC) under the Academic Strategic Alliances Program of the Accelerated Strategic Computing Initiative (ASCI/ ASAP). Additional computations were performed on the ASCI Bluemountain computer at Los Alamos National Laboratory, and its availability for this work is also gratefully acknowledged.

\section{APPENDIX A: STRUCTURE-FUNCTION RELATION USING A SPHERICAL AVERAGE}

We use the structure-function relation for LES, Eq. (18), but instead of averaging on a circle, we take the average over all possible directions of the separation vector $\mathbf{r}$ with fixed length $r$ from a given location $\mathbf{x}$. As before, the coordinate system is chosen such that the three-axis is always aligned with $\mathbf{r}$, i.e., it will rotate with $\mathbf{r}$ over all possible directions as the average is taken. The angle $\theta$ in Eq. (18) is the polar angle of the vector $\mathbf{e}_{v}$ describing the orientation of the vortex structure, cf. Eq. (7). Equation (18) does not depend on the azimuthal angle, therefore the spherical average, denoted by superscript $s$, reduces to

$$
\widetilde{F}_{2}^{s}(\mathbf{x})=2 \int_{\theta=0}^{\pi} \int_{k=0}^{k_{c}} E(k)\left[1-J_{0}(r k \sin \theta)\right] \sin \theta d k d \theta .
$$

Here, the integral over $\theta$ can be performed analytically, see, for example, Ref. 36, and we obtain

$$
\widetilde{F}_{2}^{s}(\mathbf{x})=2 \int_{0}^{k_{c}} E(k)\left[2-\pi J_{-1 / 2}\left(\frac{k r}{2}\right) J_{1 / 2}\left(\frac{k r}{2}\right)\right] d k .
$$

Using the properties of Bessel functions, ${ }^{36}$ that $J_{1 / 2}(z)$ $=\sqrt{2 /(\pi z)} \sin z$ and $J_{-1 / 2}(z)=\sqrt{2 /(\pi z)} \cos z$, this can be transformed into

$$
\widetilde{F}_{2}^{s}(\mathbf{x})=4 \int_{0}^{k_{c}} E(k)\left[1-\frac{\sin k r}{k r}\right] d k .
$$

As required for consistency, taking a spherical average over the structure-function relation for the anisotropic stretchedvortex model recovers the functional form of the structurefunction relation for isotropic turbulence, $\mathrm{cf}$. Batchelor. ${ }^{24}$

\section{APPENDIX B: ANALYTICAL APPROXIMATION FOR THE INTEGRAL IN THE STRUCTURE-FUNCTION EQUATION}

An analytical approximation for the integral in the denominator of Eq. (20) is derived so that the evaluation of this equation for $\mathcal{K}_{0} \epsilon^{2 / 3}$ can be simplified in actual simulations. The integral to be discussed is

$$
\begin{aligned}
Q(d, \sigma) \equiv & \int_{\phi=0}^{2 \pi} \int_{s=0}^{\pi} s^{-5 / 3} \\
& \times\left[1-J_{0}\left(s d \sqrt{1-\sigma \cos ^{2} \phi}\right)\right] d s d \phi,
\end{aligned}
$$

which is a function of $d \equiv r / \Delta$ and $\sigma \equiv \sin ^{2} \psi$. We first consider $d$ small. Then the whole argument of the Bessel function is small, and we use the power series expansion of $J_{0}$ truncated to second order. Substituting this in the integral and performing the integration we obtain for small $\sigma$ :

$$
Q(d, \sigma) \approx \frac{3 \pi^{7 / 3}}{16}(2-\sigma) d^{2}, \quad \text { for } d \ll 1 .
$$

For large $d$, we substitute $v \equiv s d$. Then we have for $Q$ :

$$
\begin{aligned}
Q= & d^{2 / 3} \int_{\phi=0}^{2 \pi} \int_{v=0}^{\pi d} v^{-5 / 3} \\
& \times\left[1-J_{0}\left(v \sqrt{1-\sigma \cos ^{2} \phi}\right)\right] d v d \phi .
\end{aligned}
$$

But for large $d$ we can, to a good approximation, set the upper limit of the $v$-integration to infinity. Introducing the change of variable $w \equiv v \sqrt{1-\sigma \cos ^{2} \phi}$ we obtain

$$
\begin{aligned}
& Q \approx d^{2 / 3} \int_{\phi=0}^{2 \pi}\left(1-\sigma \cos ^{2} \phi\right)^{1 / 3} d \phi \\
& \times \int_{w=0}^{\infty} w^{-5 / 3}\left[1-J_{0}(w)\right] d w .
\end{aligned}
$$

The integration over $w$ can now be carried out analytically to give

$$
C_{1} \equiv \int_{w=0}^{\infty} w^{-5 / 3}\left[1-J_{0}(w)\right] d w=\frac{\pi}{2^{2 / 3} \sqrt{3} \Gamma^{2}\left(\frac{4}{3}\right)} .
$$

The integrand in the $\phi$ integral can be expanded in a power series for $\sigma$ around $\sigma=0$, and then the integral can be performed to obtain

$$
\begin{aligned}
G(\sigma) & \equiv \int_{\phi=0}^{2 \pi}\left(1-\sigma \cos ^{2} \phi\right)^{1 / 3} d \phi \\
& \approx 2 \pi-\frac{\pi}{3} \sigma-\frac{\pi}{12} \sigma^{2}-\frac{25 \pi}{648} \sigma^{3}-\frac{175 \pi}{7776} \sigma^{4}+O\left(\sigma^{5}\right) .
\end{aligned}
$$

For large $d$ we, therefore, have the approximation:

$$
Q(d, \sigma) \approx C_{1} G(\sigma) d^{2 / 3} \text { for } d \gg 1
$$

For the implementation, we use a composite formula which blends the two asymptotic results for small and large $d$. This approximate expression for the integral, Eq. (B1), in the structure-function relation is defined as follows:

$$
Q_{\text {approx }}(d, \sigma) \equiv \frac{\left(3 \pi^{7 / 3} / 16\right)(2-\sigma) C_{1} G(\sigma) d^{2}}{C_{1} G(\sigma)+\left(3 \pi^{7 / 3} / 16\right)(2-\sigma) d^{4 / 3}} .
$$

This expression is asymptotic to Eq. (B2) for $d \ll 1$ and to Eq. (B7) for $d \gg 1$. Comparisons of this approximate relation with results from the numerical integration of the exact expression, Eq. (B1), are shown in Tables II and III. The error 
TABLE II. Comparison of the approximate relation $Q_{\text {approx }}$, Eq. (B8), with numerical integration results $Q_{\text {ref }}$ for the integral in the structure-function relation, $\sigma=\frac{1}{2}$.

\begin{tabular}{rrrc}
\hline \hline \multicolumn{1}{c}{$d$} & \multicolumn{1}{c}{$Q_{\text {ref }}$} & $Q_{\text {approx }}$ & $\left|Q_{\text {ref }}-Q_{\text {approx }} /\right| Q_{\text {ref }} \mid$ \\
\hline 1.0 & 3.37 & 2.71 & 0.196 \\
2.0 & 8.62 & 7.19 & 0.165 \\
5.0 & 19.38 & 19.26 & 0.006 \\
10.0 & 33.34 & 34.54 & 0.036 \\
20.0 & 55.49 & 57.78 & 0.041 \\
50.0 & 105.92 & 109.17 & 0.031 \\
\hline \hline
\end{tabular}

can become quite large (up to 20\%) for $d \rightarrow 1$. But in the important near-wall region one typically has $d \gg 1$ because of smaller grid spacing in the wall-normal direction in this region (i.e., $\Delta<r$, cf. Sec. III C). If the use of more precise values of the integral was desired, a two-dimensional tablelookup of precomputed values from the numerical integration of $Q$ could be used instead.

\section{APPENDIX C: APPROXIMATE SOLUTION OF THE LOCAL BALANCE EQUATION FOR THE DISSIPATION}

The balance equation (24) may be written in the form

$$
F(X)=1-\left(\Pi_{1}-3 \Pi_{4}\right) X^{4}-3 \Pi_{4} \frac{X^{14 / 3}}{J^{2 / 3}}=0, \quad X<J,
$$

where $\Pi_{4}=\Pi_{2} \Pi_{3}$. Here we briefly discuss the properties of (C1) when the parameters $\Pi_{1}$, and $\Pi_{4}$ are given. Note that $\Pi_{1} \geqslant 0$ but $\Pi_{4}$ can be of either sign. Solutions of $(\mathrm{C} 1)$ are used only when $X<J$. Otherwise the stress tensor is set to zero because $K=0$. When $\Pi_{4} \geqslant 0$, Eq. (C1) has only one real positive solution. This satisfies $X \geqslant J$ when $\Pi_{1} \leqslant J^{-4}$ and $X$ $<J$ when $\Pi_{1}>J^{-4}$. When $\Pi_{4}<0$, Eq. $(\mathrm{C} 1)$ has either two real positive solutions which we denote by $X_{A}$ and $X_{B}$, respectively, or no real solutions. The solution of physical interest, $X_{A}$ is that on the branch which is a continuation of the solution $X_{A}=\Pi_{1}^{-1 / 4}$ for $\Pi_{4}=0$. The second real solution $X_{B}$ is a bifurcation from infinity. There are no real solutions inside a region $R$ defined by

$$
0<\Pi_{1}<-3\left|\Pi_{4}\right|+\frac{7}{\left(2 J^{2 / 3}\right)^{6 / 7}}\left|\Pi_{4}\right|^{6 / 7}, \quad \Pi_{4}<0,
$$

In $\Pi_{1}-\Pi_{4}$ space the boundary of $R$ passes through the origin and the point $\left[\Pi_{1}, \Pi_{4}\right]=\left[0,-(7 / 3)^{7} /\left(2 J^{2 / 3}\right)^{6}\right]$, and has

TABLE III. Comparison of the approximate relation $Q_{\text {approx }}$, Eq. (B8), with numerical integration results $Q_{\text {ref }}$ for the integral in the structure-function relation, $\sigma=1$

\begin{tabular}{rrrc}
\hline \hline \multicolumn{1}{c}{$d$} & $Q_{\text {ref }}$ & $Q_{\text {approx }}$ & $\left|Q_{\text {ref }}-Q_{\text {approx }}\right| /\left|Q_{\text {ref }}\right|$ \\
\hline 1.0 & 2.27 & 1.94 & 0.146 \\
2.0 & 6.01 & 5.42 & 0.097 \\
5.0 & 14.45 & 15.46 & 0.071 \\
10.0 & 25.45 & 28.47 & 0.119 \\
20.0 & 42.93 & 48.25 & 0.124 \\
50.0 & 82.76 & 91.75 & 0.109 \\
\hline \hline
\end{tabular}

a turning point at $\left[\Pi_{1}, \Pi_{4}\right]=\left[J^{-4},-2 J^{-4}\right]$. When $\left[\Pi_{1}, \Pi_{4}\right]$ falls inside $R$ the stress tensor is set to zero (conditional clipping). For $J=1$ used presently for some decaying turbulence runs, this requires $\Pi_{1}<1$, and a range of $\Pi_{4}$ satisfying (C2). From (25) $\Pi_{1}$ is the order of the square of a cell Reynolds number. When $\Pi_{1}<1$ the SGS stresses are dominated by the resolved viscous stresses and clipping in a small fraction of cases has little effect on the overall calculation. Extensive experience with application of the model for $32^{3}$ box turbulence shows that the incidence of clipping events maximizes at about $2 \%$ when $R_{\lambda} \approx 27$ (marginal resolution) and are $O\left(10^{-5}\right)$ at $R_{\lambda}=O(100)$.

When $J \rightarrow \infty$ with $\nu$ finite-used for all present channel calculations- $(\mathrm{C} 1)$ can be solved analytically. For $\Pi_{1}$ $-3 \Pi_{4}>0$, the first two terms of $(\mathrm{C} 1)$ are in balance, giving $X=\left(\Pi_{1}-3 \Pi_{4}\right)^{-1 / 4}$. Then $\epsilon$ and $\mathcal{K}_{0}$ can be calculated because $\mathcal{K}_{0} \epsilon^{2 / 3}$ is known. The subgrid-kinetic energy $K$ follows from (21) and does not require $X$. When $\Pi_{1}-3 \Pi_{4}<0$, the second and third terms are in balance, giving, for large $J$,

$$
X \approx J\left(\frac{3 \Pi_{4}-\Pi_{1}}{3 \Pi_{4}}\right)^{3 / 2} .
$$

When $J \rightarrow \infty$ then $X \rightarrow \infty$ and the local resolved dissipation and the backscatter are in exact balance. A short calculation then shows that

$$
K=\frac{2 \nu \widetilde{S}_{i j} \widetilde{S}_{i j}}{\widetilde{S}_{i j}\left(\delta_{i j}-e_{i}^{v} e_{j}^{v}\right)}
$$

and $\tau_{i j}$ can be evaluated. The line $\Pi_{1}-3 \Pi_{4}=0$ is a discontinuity in $X$ but it is straightforward to show that $\tau_{i j}$ remains continuous.

When $J$ is finite, the above expressions for $X$ can be used as the zeroth order basis for either linear or quadratic expansions giving approximate analytical solutions to (C1). These can be used directly or as initial approximations for numerical solution of (C1) by Newton's method. This is straightforward.

${ }^{1}$ M. Lesieur and O. Métais, "New trends in large-eddy simulations of turbulence,"' Annu. Rev. Fluid Mech. 28, 45 (1996).

${ }^{2}$ U. Piomelli, "Large-eddy simulation: Achievements and challenges," Prog. Aerosp. Sci. 35, 335 (1999).

${ }^{3}$ M. Germano, U. Piomelli, P. Moin, and W. H. Cabot, "A dynamic subgrid-scale eddy viscosity model," Phys. Fluids A 3, 1760,3128(E) (1991).

${ }^{4}$ D. K. Lilly, "A proposed modification of the Germano subgrid-scale closure method," Phys. Fluids A 4, 633 (1992).

${ }^{5}$ S. Ghosal, T. S. Lund, P. Moin, and K. Akselvoll, "A dynamic localization model for large-eddy simulation of turbulent flows," J. Fluid Mech. 286, 229 (1995).

${ }^{6} \mathrm{U}$. Piomelli and J. Liu, "Large-eddy simulation of rotating channel flows using a localized dynamic model,'” Phys. Fluids 7, 839 (1995).

${ }^{7} \mathrm{O}$. Métais and M. Lesieur, "Spectral large-eddy simulation of isotropic and stably stratified turbulence,"' J. Fluid Mech. 239, 157 (1992).

${ }^{8}$ E. Lamballais, O. Métais, and M. Lesieur, "Spectral-dynamic model for large-eddy simulations of turbulent rotating channel flow," Theor. Comput. Fluid Dyn. 12, 149 (1998).

${ }^{9}$ F. Ducros, P. Comte, and M. Lesieur, "Large-eddy simulation of transition to turbulence in a boundary layer developing spatially over a flat plate,"' J. Fluid Mech. 326, 1 (1996).

${ }^{10} \mathrm{P}$. Comte, J. H. Silvestrini, and P. Begou, "Streamwise vortices in largeeddy simulation of mixing layers," Eur. J. Mech. B/Fluids 17, 615 (1998).

${ }^{11}$ A. Leonard, "Energy cascade in large-eddy simulations of turbulent fluid 
flows," in Advances in Geophysics, edited by F. N. Frankiel and R. E. Munn (Academic, New York, 1974), Vol. 18A, pp.237-249.

${ }^{12}$ A. Leonard, "Large-eddy simulation of chaotic convection and beyond," AIAA 97-0204, 1997.

${ }^{13}$ B. Kosović, "Subgrid-scale modelling for the large-eddy simulation of high-Reynolds-number boundary layers,"’ J. Fluid Mech. 336, 151 (1997).

${ }^{14}$ J. A. Domaradzki and E. M. Saiki, "A subgrid-scale model based on the estimation of unresolved scales of turbulence," Phys. Fluids 9, 2148 (1997).

${ }^{15}$ J. A. Domaradzki and K.-C. Loh, "The subgrid-scale estimation model," in Recent Advances in DNS and LES, edited by D. Knight and L. Sakell (Kluwer Academic, Boston, 1999), pp. 121-131.

${ }^{16}$ J. A. Domaradzki and K.-C. Loh, "The subgrid-scale estimation model in the physical space representation," Phys. Fluids 11, 2330 (1999).

${ }^{17} \mathrm{~S}$. Ghosal and P. Moin, "The basic equations for the large eddy simulation of turbulent flows in complex geometry," J. Comput. Phys. 118, 24 (1995).

${ }^{18}$ D. I. Pullin and P. G. Saffmann, "Reynolds stresses and one-dimensional spectra for a vortex model of homogeneous anisotropic turbulence," Phys. Fluids 6, 1787 (1994).

${ }^{19}$ A. Misra and D. I. Pullin, "A vortex-based subgrid stress model for largeeddy simulation," Phys. Fluids 9, 2443 (1997).

${ }^{20}$ A. Misra, D. I. Pullin, and D. C. Chan, "Large-eddy simulation using the stretched-vortex SGS model," in Advances in DNS/LES, edited by C. Liu and Z. Liu, Proceedings of the First AFOSR International Conference on DNS/LES (Greyden, Columbus, 1997), pp. 385-392.

${ }^{21}$ T. S. Lundgren, "Strained spiral vortex model for turbulent fine structure," Phys. Fluids 25, 2193 (1982).

${ }^{22} \mathrm{~S}$. Kida and Y. Murakami, "Kolmogorov similarity in freely decaying turbulence,' Phys. Fluids 30, 2030 (1987).

${ }^{23}$ S. G. Saddoughi and S. V. Veeravalli, "Local isotropy in turbulent boundary-layers at high Reynolds-number," J. Fluid Mech. 268, 333 (1994).
${ }^{24} \mathrm{G}$. K. Batchelor, The Theory of Homogeneous Turbulence (Cambridge University Press, Cambridge, UK, 1953).

${ }^{25}$ C. Canuto, M. Y. Hussaini, A. Quarteroni, and T. A. Zang, Spectral Methods in Fluid Dynamics (Springer-Verlag, New York, 1988).

${ }^{26}$ S. K. Lele, "Compact finite difference schemes with spectral-like resolution," J. Comput. Phys. 103, 16 (1992).

${ }^{27}$ R. Schiestel and S. Viazzo, "A Hermitian-Fourier numerical method for solving the incompressible Navier-Stokes equations," Comput. Fluids 24, 739 (1995).

${ }^{28}$ D. C. Chan, "Effects of rotation on turbulent convection: Direct numerical simulation using parallel processors," Ph.D. thesis, University of Southern California, 1996.

${ }^{29} \mathrm{~A}$. N. Kolmogorov, "Local structure of turbulence in an incompressible viscous fluid at very high Reynolds numbers," Dokl. Akad. Nauk SSSR 30, 229 (1941).

${ }^{30} \mathrm{G}$. Comte-Bellot and S. Corrsin, "Simple Eulerian time correlation of fulland narrow-band velocity signals in grid-generated, 'isotropic' turbulence,"' J. Fluid Mech. 48, 273 (1971).

${ }^{31}$ R. D. Moser, J. Kim, and N. N. Mansour, "Direct numerical simulation of turbulent channel flow up to $\operatorname{Re}_{\tau}=590$,' Phys. Fluids 11, 943 (1999).

${ }^{32}$ J. Kim, P. Moin, and R. Moser, "Turbulence statistics in fully developed channel flow at low Reynolds number," J. Fluid Mech. 177, 133 (1987).

${ }^{33} \mathrm{~T}$. Wei and W. W. Willmarth, "Reynolds-number effects on the structure of a turbulent channel flow," J. Fluid Mech. 204, 57 (1989).

${ }^{34}$ AGARD, AR-345, A selection of test cases for the validation of largeeddy simulations of turbulent flows, 1998.

${ }^{35}$ P. Moin and J. Kim, "Numerical investigation of turbulent channel flow," J. Fluid Mech. 118, 341 (1982).

${ }^{36}$ I. S. Gradshteyn and I. M. Ryzhik, Table of Integrals, Series, and Products, 5th ed. (Academic, San Diego, 1994). 